\title{
The emergence of governance norms in volunteer- driven open source communities
}

\author{
Mirko Boehm ${ }^{a}$ \\ (a) Visiting Lecturer, Chair of Innovation Economics, Technical \\ University of Berlin; Director, Linux System Definition, Open \\ Invention Network
}

DOI: 10.5033/jolts.v11i1.131

\begin{abstract}
Free and open source software communities develop their governance norms and practises as they grow from small to medium to large sized social groups. Communities with a small number of participants typically organise informally. As the community grows, the need for coordination grows as well and at some point, a more structured organisation becomes necessary.

The growth stages are defined by the coordination mechanisms applied - ad-hoc coordination for the initial small group, consensus focused auto-organisation for the medium sized group, and structured, more formalised coordination for the large sized group.

The main interest of the communities is to attract and retain contributors and to facilitate contributions to their products. The communities studied in this qualitative embedded multiple-case study, exhibit governance related debates and conflicts, as they reached a large size, leading to difficulties in further growing the number of involved contributors and sustaining the community activities.

The paper researches the emergence of governance norms in these communities and the role these norms, once established, play in the management of the communities in their then current stage.

The study finds that the governance norms in communities are commonly developed by participants that do not think them necessary, for a community that does not want them at the time. The result is frequently implicit, under-documented norms that increase barriers to entry for newcomers and allow incumbent contributors the instruments to derail unwanted decisions.

The paper focuses on the essential contradiction between the communities' aim to maintain devolved authority at the contributor level and a requirement for effective decision making and policing mechanisms to implement and maintain that. It recommends that communities, instead of deferring or down-playing the need to set up explicit governance norms, purposefully develop norms that explicitly define structure and processes so that they support, enforce and protect the devolved authority their participants should have and encourages new participants.
\end{abstract}




\section{Keywords}

Free and Open Source Software; governance; code of conduct; meritocracy; FSFE; KDE Community; Wikimedia

\section{An inside view on social norms in communities}

On February 3, 2016, something happened in the KDE free and open source software (FOSS) community that would become the dominant topic of discussion for more than 6 months: An announcement ${ }^{1}$ was sent to the community mailing list that a draft of a new vision for the community was being worked on. This announcement triggered close to 350 postings to various mailing list threads, constituting almost half of all discussions within the community in the first half of 2016. It led to heated discussions between drafters of competing visions, public endorsements and statements of support, virtual ad-hominem attacks and even contributors leaving the community in anger. How could an announcement of something so basic, so fundamental to a large decentralised group of volunteers, like a vision, create such distress?

In May 2016, a code of conduct for the Free Software Foundation Europe (FSFE) was announced to the organisation's coordinators. After small changes, a version was approved and sent to the core team for a decision to be made in June. After just a few people voiced an opposition to some of the wording, the process came to a halt. When it was picked up again in October of the same year and circulated in an almost unchanged form to the same people who had seen it already in May-June, it spawned one of the fiercest debates in the history of FSFE with more than 200 mails in just two weeks. Suddenly, people spoke up in opposition not only to the content of the code of conduct but about the very need for a code of conduct in general. Indeed, the code of conduct had, in the eyes of some of the participants, become a tool not to include contributors but to silence unwanted opinions. While the general consensus seems to have been in favour of adopting a code of conduct, the process came to a halt again, since no decision could be reached. The code of conduct was finally adopted without substantial changes in October 2017.

There are many instances of such soul-searching in FOSS communities as they reach maturity and achieve a large number of contributors. It can be observed that these controversies focus on questions regarding how the communities internally manage their social norms and questions of community governance, which form the totality of implicit and explicit behavioural norms, codes and processes that regulate the relationship between contributors and the community. While these are certainly not the only challenges the communities face as they grow, evolving community governance appears to be a particularly difficult problem for each community to manage.

When interpreting the habits and practises of voluntary contributor collaboration in FOSS communities as a cultural phenomenon, governance norms are seen as the inside view on the culture of that particular community. They are an outward expression of the way the communities see themselves. Understanding this inside view as to how the communities are expected to operate is relevant not only regarding issues of community management, but also to outsiders as the basis of the views held by that community's contributors and how to engage with that community. To establish successful collaboration with these communities, the public, regulators, businesses and influencers of technical innovation such as standards development organizations (SDOs) or the patent offices would be well-advised to understand the cultural norms and practises of these FOSS communities.

This paper researches the governance norms that have evolved in volunteer-driven FOSS communities as they grow from an initiative of a few contributors to large and often international organisations. Assuming that these norms are based on the aggregate of the individual convictions

1 https://mail.kde.org/pipermail/kde-community/2016q1/002241.html 
and expectations of those contributing to the community, the paper describes this inside view that the communities have of themselves and in particular the behaviour the actors engaged in the community expect from their fellow contributors, from the community as a whole and from outsiders - individuals, other organisations and the public.

\section{Governance in communities with voluntary participation}

For this study, the work of the wider Open Source community is primarily viewed as a social process producing information as a public good. It is a knowledge-intensive process that has inputs in the form of labour (the work of the contributors) and capital (the funding required by the communities). The output is information goods, most prominently the software components that are freely distributed to the public. The nature of free software licenses makes them "non-excludable" and "non-rivalrous" and therefore "public goods".

The production of a public information good is one key element that defines a FOSS community. The other key element is voluntary participation of the contributors in the community. The understanding of FOSS community applied in this paper is that of a social group of contributors that participate voluntarily in the production of public information goods. ${ }^{2}$ The participants in these groups, the contributors (see section 2.2), collectively create the community's products and make them available to the general public by distributing them under a free or open source software license. This study focuses on communities that consist predominantly of individual volunteer contributors.

Communities that grow beyond a very small group of contributors develop (sometimes unconsciously) functional specialisation between their contributors, division of labour between formally and informally defined subgroups, and integration of the individual contributions into an overall product. They become organisations. Functions that contributors specialise in can be product related (software development or content creation in general, "maintainership" over submodules, or release management) or support (marketing and public relations, finance, event management and legal). To successfully release products over time, communities need to coordinate the work of the individual contributors so that, through a repetitive process of content creation, gatekeeping and filtering for quality, integration and distribution, the product improves over time. ${ }^{3}$ Coordination in this context is understood as a process, not as a task performed by a manager. With respect to the production process, the need for community governance results from the necessity to coordinate the work of a diverse group of volunteers to create the community product.

From an outside perspective, of users or the general public, the communities are mainly known for the products they create. Potential contributors want to engage with the community based on the product related participation opportunities, and on what is generally known about the culture of the community. A common recommendation is to "treat every user as a potential volunteer". ${ }^{4}$ Most contributors participate in a community for a limited period of time, leading to fluctuation in participation. To grow the number of incoming contributions, communities need to attract new contributors and retain the existing ones, so that the difference between influx and outflow remains positive. With regard to the interaction with the outside world, the need for community governance results from the necessity to maintain and grow the contributor base that forms the community.

Even though FOSS communities commonly operate as decentralised self-organised groups, they develop elaborate informal and formal rules and practises for their social process. These rules and

2 Albert O. Hirschman. The Passions and the Interests. 20 Anv Sub. Princeton University Press, Jan. 1997.

3 Yochai Benkler. "Coase's Penguin, or, Linux and "The Nature of the Firm"”. In: The Yale Law Journal 112.3 (Dec. 2002), pp. 369+.

4 Karl Fogel. Producing Open Source Software: How to Run a Successful Free Software Project. O'Reilly Media, Inc., Oct. 2005 . 
practises are referred to as the governance norms of the community. A social norm is "a prescribed guide for conduct or action which is generally complied with by the members of a society". ${ }^{5}$ The term governance "refers to all processes of social organisation and social coordination" ${ }^{6}$ within groups. It describes the processes of governing a formal or informal organisation performed by a formal government, a market or a network. Governance is expressed through a wide range of instruments ranging from laws to social norms, as well as language and culture. Any social group that coordinates working together towards a common goal will exhibit some form of governance. Whereas government refers to the institutions that exert power and influence over a constituency, governance can exist without institutions. Communities often hesitate to develop formal governing structure. This directs the focus of analysis on governance (a process) over government (the structure) when studying FOSS communities.

The subject of governance can be considered to be decision making and conflict resolution within the social group, by using broad definitions for both terms. "Decisions" is used here in the sense that whenever a small subset of the contributors or the whole community jointly agree on a course of action on some subject, a decision is made. Similarly, "conflict" is understood broadly as any disagreement of one or a group of contributors with any action or decision made by another subgroup or the community as a whole. Decisions do not need to be made in a formalised process, nor does conflict require a formal complaint or a heated argument. Decision making and conflict resolution are essential elements of collective action. ${ }^{7}$ How the organisation defines who may participate in what decisions as a community member, and what organs form the organisational structure, characterises key aspects of that governance.

It can be assumed that organisations exist to further the common interests of their members. ${ }^{8}$ The reason for FOSS communities to exist is to facilitate the interests and motivations of their contributors. To illustrate the governance of FOSS organisations, this study will review the reasons why the organisations exist, the organisational structure of the community, the processes by which decisions are made and challenged, and how and with which roles contributors participate in them.

The ethics and convictions of the individual contributors should be reflected in the organisation's vision and mission statements. The formal and informal organisational structure provides the framework for the community's production process. Constitutional documents like bylaws and manifestos establish formal structure. Representative bodies like boards, committees and working groups are the most visible formalised form of it. Formal structure projects authority by assigning decision making power to individuals or organisational units. In addition to those, informal structures that are more difficult to identify are likely to exist. Informal structure manifests itself in decisions that bypass hierarchy, or in strong impact of the opinions of individuals that are not appointed to representative positions. FOSS communities commonly show a preference for minimal formal organisation (see section 2.4), which leads to the assumption that informal structure has a more preferable effect than usual. Formal organisation is also more difficult to change, since it typically requires both a qualified majority of the group members and a conscious effort to understand and reconsider the current structure and identify how it should be changed.

One potential reason for a perceived need for organisational change is a divergence between the formal and informal structure. Opposition to reform indicates that group members may be more comfortable with the existing balance of formal and informal structure. Decision making processes and conflict resolution mechanisms define how decisions are initiated and then made, and how to appeal against or escalate them in cases of disagreement, how decisions will be implemented or enforced, and how the community deals with minority opinions and opposition, especially in the case

5 Edna Ullmann-Margalit. The emergence of norms. Clarendon Press, 1977.

6 Mark Bevir. Governance - a very short introduction. Oxford University Press, 2012.

7 Russell Hardin. Collective Action. RFF Press, June 1982.

8 Olson Mancur. The Logic of Collective Action: Public Goods and the Theory of Groups. Revised. Harvard economic studies, v. 124. Harvard University Press, Jan. 1965. 
of controversial decisions.

Decision making processes correspond to organisational structure in that commonly, paths of escalation or appeal follow the hierarchy of formal organisation. Conflict resolution is directly related to decision making processes as the cause of a conflict is either the wish for a decision to be made, or to appeal against one that was made. The balance between decision making processes, instruments of appeal and conflict resolution is what enables contributors to influence the community production process.

The social order within the community defines which stakeholders can take part in what group decisions. Differentiation can for example be based on eligibility or group status. A regular contributor may not be eligible to take part in a board decision or may not have the status to take over a maintainer role. The question of social order in communities boils down to what decisions a contributor can participate in, and what the impact of the individual vote is. It relates to the definition of group membership that separates insiders from outsiders, but also possibly to the status of groups within the community. It is also related to how contributions are valued and translate into merit and recognition for the contributor. There may be a sense of equality, or a sense of elitism where "only the core contributors should have a say". If social order in communities is considered important, there should be well-defined processes on how to gain access to those status groups within the community that carry weight in important decisions.

FOSS communities sometimes discount the importance of decision-making or claim that decisions are made or conflicts resolved by "the wider community", and that therefore organisational theory does not apply. This argument however does not hold water, since it cannot reasonably be disputed that communities delimit members from outsiders, have status groups, make decisions and resolve conflicts (even if those elements are not all made explicit). ${ }^{9}$ By analysing organisational structure, decision making processes and the community social order as key elements of governance, it is possible to compare communities even if they create unrelated products.

\subsection{Growth stages of communities}

The differentiation between the inside and the outside view of the community's social process puts the emphasis on the demarcation of the social group, in as much as it defines who is a member of the group and who is an outsider. Being an insider means accepting the group's rules, providing influence and in turn expecting to participate in the group's governance. Being an outsider leaves a choice to either interact with the community and accepting its norms, or to abstain from interacting with it. The community is afforded the same choice not to engage with an outsider based on how compatible their actions are with the group's norms.

Since about 2010, participation in FOSS activities as a phenomenon has changed from an exotic movement to a common mode of operation in the ICT industry. ${ }^{10}$ This suggests that communities have also matured into established organisations with solidified cultural norms and values. The communities studied in this report have all existed for longer than a decade. They will be viewed as mature and stable organisations where processes can be observed through the activities within their formal and informal structure. Their norms and values have developed over time as a result of the interaction between community participants who join the group voluntarily out of their own motivations, and the community as an organisation of its own, which creates structure and processes according to the goals of the group and the strategies chosen to reach them.

9 Amitai Etzioni. "Two Approaches to Organizational Analysis: A Critique and a Suggestion”. In: Administrative Science Quarterly 5.2 (1960), pp. 257-278.

10 Jeff Licquia and Amanda McPherson. A \$5 Billion Value: Estimating the Total Development Cost of Linux Foundation's Collaborative Projects. Tech. rep. https://www.linux.com/publications/estimating-total-development-cost-linuxfoundations-collaborative-projects. The Linux Foundation. 
The communities will be investigated at three different growth stages: The point of foundation called the initial stage, the time when the group has reached a small to medium number of contributors (typically between 20 and 50 active contributors) called the medium stage, and a late stage with a large number of community members (often more than 100). The growth stages are defined by the coordination mechanisms applied to the social process, which show different characteristics in these different stages of development.

At the time that a particular FOSS initiative is formed and in its initial stage the goals and motivations of the group of founders and of the initiative as a whole are identical. There is commonly great enthusiasm about the joint initiative. The original authors publish their work and communicate that contributions from others are welcome and appreciated. More contributors join and participate out of a motivation similar to the motivations of the original authors - to contribute to the product, make it available to the public under a FOSS license, and rely on the community to keep the process going. As long as the group is small enough for ad-hoc coordination, the subsequent contributors joining will find themselves in a similar situation. It can be assumed that the participants in the initial stage will be homogenous in their motivations, cultural backgrounds and interests. Worries about governance usually do not exist. ${ }^{11} 1213$

Interests and motivations will start to diverge as the community grows and matures. The group will reach the medium stage when the number of participants becomes too large for ad-hoc coordination and changes into a form of consensus focused auto-organisation. At this stage, deviations between individual expectations and community behaviour exist. Instead of relying on formal structure in the organisation, the communities rely on a consensus-driven, participative debate culture. Disagreements will be discussed at length until a resolution is achieved. The resolution does not necessarily require consensus or a formal decision.

The KDE community, for example, applies a method called "lazy consensus", in which contributors have begun to work on their favoured solution while alternative courses of action are still being discussed. The direction the community later prefers can then be decided based on the results of the discussion and on the experience from the work already provided by its contributors. Other communities apply similar mechanisms that prefer product related contributions over "bureaucracy". It is apparent that such mechanisms rely on close cohesion of the group's participants, a low grade of specialisation amongst the contributors and a relatively small number of stakeholders in the decisions. Not only are the communities themselves content with such informal self-coordination, they also develop a strong preference for the absence of formal structure. Since contributors participate voluntarily, they feel entitled to self-identification of tasks and to work free from direction given by others. ${ }^{14}$ While it may cause friction, self-identification contributes to the allocation efficiency of peer-production processes. ${ }^{15}$

The transition into the late stage of community development is commonly marked by more formalisation. Communities may establish internal working groups to facilitate contributions to more specialised topics. To coordinate with external partners, they may nominate community members to represent the community in their committees. To account for these delegated responsibilities, the representatives may be required to report on their work on a frequent basis at regular meetings. In general, more functional differentiation occurs between the community participants. Delegation of power and responsibility becomes more pronounced, leading to a more prominent role for the

11 Eric S. Raymond. The Cathedral and the Bazaar: Musings on Linux and Open Source by an Accidental Revolutionary (O'Reilly Linux). O’Reilly, Oct. 1999.

12 Karim Lakhani and Robert G. Wolf. "Why Hackers Do What They Do: Understanding Motivation and Effort in Free/Open Source Software Projects”. In: Social Science Research Network Working Paper Series (Sept. 2003).

13 Steven Weber. The Success of Open Source. Harvard University Press, Apr. 2004.

14 Karl Fogel. Producing Open Source Software: How to Run a Successful Free Software Project. O’Reilly Media, Inc., Oct. 2005.

15 Yochai Benkler. "Coase's Penguin, or, Linux and "The Nature of the Firm"”. In: The Yale Law Journal 112.3 (Dec. 2002), pp. 369+. 
community leaders.

At this point leadership positions that previously more or less fell to those who volunteered to speak to the press or be elected to the board become more prestigious. Appointments carry more weight and elections for them grow competitive. The differentiation of roles within the community enables jockeying for position and a sense of entitlement, especially as regards long-standing contributors. Once this formal organisational structure is established, the community shows behavioural patterns similar to other larger common good oriented community organisations like unions, sports clubs or cultural initiatives. Being a part of the community becomes a motivation in its own right, complementing the motivation to contribute to the community product directly. Matters of procedure and community management attract more attention. A share of the collective energy of the community is redirected inwards to discuss the community itself. At the same time, behavioural norms are still in place that developed during the early and medium stage. For example, communities have established a "break all the rules" rule that postulates that every participant is free to decide the best course of action, even if it means ignoring a norm or rule. Or there may be a "who does the work decides" rule which postulates that those who take part in the debate should not interfere with those working directly on the community product.

Based on these considerations, it can be expected that communities in the initial stage require almost no coordination, communities in the medium stage rely on organic self-coordination, and communities in the late stage act more in accordance with the logic of collective action in large groups.$^{16}$ The transition into the late stage should necessitate a change of the effective community governance norms away from informal mechanisms of the medium stage towards more explicit, formal mechanisms appropriate for larger-scale collective provision processes. The intense governance-related conflicts and debates that accompany the shift of the communities into the late stage indicate that this change did not fully happen in the cases studied in this report.

Intense inner social conflicts indicate a divergence between the individual expectations of contributors and the group norms developed by the community. These conflicts may be resolved positively, resulting in a re-alignment of individual and group motivation. However, if the conflict is too severe or for other reasons cannot be resolved satisfactorily, it may also lead to either individual contributors deciding not to participate in the group anymore, or the conflict may cause a fork, where the group splits into two that continue to develop towards the initial goal separately ${ }^{17}{ }^{17}$ Forks are rare, as substantial effort must be invested to create a competing community organisation. More commonly, contributors defect if their perception of the quality of the community diminishes. Since there is no centralised resource planning, defections may go unnoticed. It is difficult to assess the impact of individual decisions or the design of decision-making processes on the contributor base. Sometimes communities prefer not to make any decisions to avoid losing contributors, which results in indecision manifested for example in bike-shedding debates. ${ }^{18}$

\subsection{Community composition}

Entities participating in FOSS initiatives can be either individual volunteers, organisations (participating directly or through contributions of their employees) or staff employed by the community. This mix is referred to as community composition. Most communities consist of individual volunteers and employees of businesses, with a very small share of employed staff. ${ }^{19}$

16 Olson Mancur. The Logic of Collective Action: Public Goods and the Theory of Groups. Revised. Harvard economic studies, v. 124. Harvard University Press, Jan. 1965.

17 Gregorio Robles and Jesu's M. Gonz'alez-Barahona. "A Comprehensive Study of Software Forks: Dates, Reasons and Outcomes”. In: Open Source Systems: Long-Term Sustainability. Ed. by Imed Hammouda et al. Berlin, Heidelberg: Springer Berlin Heidelberg, 2012, pp. 1-14.

18 Karl Fogel. Producing Open Source Software: How to Run a Successful Free Software Project. O’Reilly Media, Inc., Oct. 2005.

19 Dirk Riehle et al. "Paid vs. Volunteer Work in Open Source". In: System Sciences (HICSS), 2014 47th Hawaii International Conference on. IEEE, Jan. 2014, pp. 3286-3295. 
This study focuses on communities that are made up predominantly by individual volunteer contributors. These communities, like KDE, may distinguish between contributions of time and effort spent by an individual contributor and the financial contributions made by businesses. The individual contributors are able to become personal members in KDE e.V. even if they contribute during work time. The businesses employing them may only gain "supporting membership" through which they support funding the organisation by paying a membership fee, but do not attain a vote on a board or in the annual general assembly.

FSFE similarly does not allow other organisations to take part in their activities directly. These rules underline the significance "volunteer driven" communities associate with individual contributions and their aversion towards any form of institutional investment. This norm of only valuing individual contributions builds upon the expectation that the community production process should be steered with regard to product quality alone, and not influenced by interests of external parties. In the case of KDE e.V., this norm is explicitly codified in the bylaws of the organisation, which only accepts individuals as members, not legal entities.

In communities with a majority of contributors who are employed by businesses, like the Linux kernel developer community, the reputation of companies is more closely related to the aggregated contributions of their employees. ${ }^{20}$ Businesses and individuals participate in FOSS activities for different reasons. Individual volunteers are mainly intrinsically motivated through a sense of achievement and personal enjoyment. Signalling of key skills to potential employers also plays a role. $^{21}$ Businesses on the other hand, are motivated by economic rewards and the opportunity to influence. For example, participation gives them the opportunity to create non-differentiating components in their products in collaboration with other parties with similar interests at drastically reduced research and development costs, as well as participation transaction cost. ${ }^{22}$ Businesses also benefit from their FOSS activities being a source of quality staff and promoting a healthy innovation ecosystem.

Depending on community composition, the communities develop norms and principles that reflect the specific mix of motivations of their constituency. This opens up a continuum with purely volunteer driven communities on one end, purely business driven communities on the other end, and mixed or hybrid communities in between.

The majority of FOSS communities are hybrids, resulting in a set of norms and practises within those communities that reflect the motivation of both organisational and individual contributors. ${ }^{23} \mathrm{We}$ expect that the norms and principles adopted by the communities can be clustered based on the contributor composition, and that communities with relatively similar contributor structures develop relatively similar norms and practises. To facilitate separate analysis of these sets of motivations, this paper focuses on studying communities that are (almost) exclusively made up of individual volunteer contributors. These communities would be expected to have developed comparable governance norms.

20 Jonathan Corbet and Greg Kroah-Hartman. Linux Kernel Development, 25th Anniversary Edition. Tech. rep. http://go.linuxfoundation.org/linux-kernel-development-report-2016 . Linux Foundation.

21 Karim Lakhani and Robert G. Wolf. "Why Hackers Do What They Do: Un- derstanding Motivation and Effort in Free/Open Source Software Projects”. In: Social Science Research Network Working Paper Series (Sept. 2003).

22 Johan Lin aker et al. "How Firms Adapt and Interact in Open Source Ecosys- tems: Analyzing Stakeholder Influence and Collaboration Patterns". In: Re- quirements Engineering: Foundation for Software Quality: 22nd International Working Conference, REFSQ 2016, Gothenburg, Sweden, March 14-17, 2016, Proceedings. Ed. by Maya Daneva and Oscar Pastor. Cham: Springer Inter- national Publishing, 2016, pp. 63-81.

23 Sonali K. Shah. "Motivation, Governance, and the Viability of Hybrid Forms in Open Source Software Development". In: Management Science 52.7 (2006), pp. 1000-1014. 


\subsection{Separation of open source products and community processes}

FOSS communities create freely available products in a social process of peer production. ${ }^{24}$ While it is a common expectation that producing a product under a free software license goes hand-in-hand with applying a transparent, open process, based on voluntary participation, this is not always the case. ${ }^{25}$ There are FOSS products that are produced by a single vendor in a closed process and without relevant participation of other parties. ${ }^{26}$ Some products are developed by a single, dominant commercial vendor where outside participants are required to grant rights to relicense the product proprietarily to the commercial vendor through some form of contribution agreement. These agreements do not reduce the freedoms provided by the product license, but they change the community process from decentralised to centralised. ${ }^{27}$

Other products, like the Linux kernel, are built by a decentralised community and do not require any attribution of rights. The licensing of FOSS products on the one hand and the community processes applied to produce them on the other need to be considered separately. While the choice of license defines whether or not a product is free or open source software, the governance norms applied by a community determine openness. ${ }^{28}$

We assume that the preference in a community for a more or less open governance model correlates closely with community composition (see section 2.2), and that volunteer driven development communities have a strong preference towards openness and transparency in their governance.

The two main schools of thought about the essence of FOSS represent these two aspects separately as well:

Some proponents of the term "open source" put more significance on whether or not a product is distributed under a FOSS license approved by the Open Source Initiative. They see software released under a free license as a means to an end.

Others who put more emphasis on software freedom consider the work of communities to be part of a political movement representing a cultural shift that works towards a world without proprietary software, with an ethical underpinning. The FSF for example argues that "software should not have owners". ${ }^{29}$ The separate product and process aspects of FOSS however are present and relevant in both schools of thought.

\subsection{Voluntary participation and meritocracy}

Both camps agree that contributors form the community by taking part in the production process voluntarily and without direct compensation for their efforts. Communities with a small number of contributors are typically organised in an informal way and work coherently. As the number of contributors grows, the difficulties of informal organisation grow until they reach a level that requires a more formal structure. There is however no authority in a position to impose such a structure.

The raison d'etre of enterprises and institutions is commonly defined ex-ante by, for example,

24 Yochai Benkler. "Coase's Penguin, or, Linux and "The Nature of the Firm"”. In: The Yale Law Journal 112.3 (Dec. 2002), pp. 369+.

25 Josh Lerner and Jean Triole. "The Simple Economics of Open Source”. In: National Bureau of Economic Research Working Paper Series (Mar. 2000), pp. 7600+.

26 Dirk Riehle. "The single-vendor commercial open course business model". In: Information Systems and E-Business Management (Nov. 2010), pp. 1-13.

27 Karl Fogel. Producing Open Source Software: How to Run a Successful Free Software Project. O’Reilly Media, Inc., Oct. 2005.

28 Liz Laffan. “A New Way of Measuring Openness: The Open Governance Index”. In: Technology Innovation Management Review 2 (2012), pp. 18-24.

29 Richard M. Stallman and Lawrence Lessig. Free software, free society: selected essays of Richard M. Stallman. SoHo Books, 2010. 
investors, regulation or government, which act as a form of higher power that imposes a purpose on the entity.

Like sovereign states, the question of the purpose of a FOSS community is self-referential. A community exists to serve the interests of the participants, which participants are also the community. Where states resort to postulating a constitution which then anchors acts of government, communities develop governance mechanisms based on voluntary participation and meritocracy (unlike in the original satirical understanding of meritocracy, the wider Open Source community historically uses the term with an overall positive connotation, which has recently been challenged). ${ }^{30}$

Voluntary participation in the group means that an acceptance and implementation of group decisions needs to be achieved based on a common and mutual understanding. Since they are contributing voluntarily, participants expect to have peer status in the group and influence according to the value of their contributions. This is what FOSS communities refer to when they call themselves meritocratic. When formal structure emerges, the principles and norms applied typically reflect voluntary participation and meritocratic peer status as well. From this, two important collective action issues are derived that communities struggle with, that of decision making, and that of enforcing conformity to social norms.

Decision making is difficult as the winning majority has no instrument to force those who disagree with the decision to implement it. There is no individual cost involved in simply ignoring a community decision. Communities therefore prefer to reach consensus and do so in a discussion process that may be laborious to reach a decision, even if only with a very qualified majority.

They intentionally refrain from allowing the same question to be re-raised after a decision is made, without reason or a great deal of effort from the participant re-raising it. Some communities explicitly acknowledge the difficulty of making formal decisions and relegate them to the status of opinion polls (Wikimedia) or restricting the use of votes to the acceptance of new members (KDE).

The sensitivity of making decisions that are not based on consensus reflects the importance of attracting and retaining contributors and underlines the social process aspect of community activity. This sometimes results in a separation of administrative leadership and product related decision making. For example, KDE e.V. manages KDE's assets and funds, but by way of a community principle may not interfere with product related technical decisions.

Mechanisms that aim to enforce conformity to social norms are mostly absent in communities. Initially most behavioural norms develop informally. In the medium and late stages, community manifestos or a code of conduct may be put in place. At this stage, the necessity for a formal rule that restricts how community members may behave may be questioned. For example in KDE and FSFE.

The communities studied did not build effective means of actively influencing behaviour towards the expected outcomes. While in early stages this need is mitigated by the strong cohesion of the group, in later stages the lack of it is often seen as an obstacle to developing more diversity. ${ }^{31}$ Based on anecdotal evidence from the interviews, the necessity for explicit behavioural rules is typically questioned by long-standing community members that are part of the dominant social group within the community. The aversion to enforcement of rules is related to the self-referential nature of communities. Critics of explicit rules often question where the authority would come from to enforce them.

30 Michael Dunlop Young. The rise of the meritocracy, 1870-2033: The new elite of our social revolution. Vol. 85. Random House, 1959.

31 http://rachelnabors.com/2015/09/01/code-of-conduct/, but also https://modelviewculture.com/pieces/a-code-of-conductis-not-enough 
Governance based on voluntary participation and meritocracy is an essential attribute of volunteerdriven communities and stems from the self-referential nature of the community's purpose. While communities that are led by a "self-appointed benevolent dictator for live" exist, the cohesion of these communities depends on this individual maintaining a strong meritocratic status. ${ }^{32}$ Separating product outputs from community process can also provide guidance as to which FOSS producing organisations should be considered communities: a community distributes products that through applying a FOSS license are public goods and the associated organisation will create this product in a process that is based on voluntary participation.

When applying these criteria to classifying organisations into FOSS communities and others, the cases studied in this report meet this requirement. A single entity that produces FOSS without voluntary participation of others, like Google developing Android, is a valuable FOSS contributor, but not a community.

\subsection{Study design and method}

The aim of this study is to describe and understand in detail how and what influences the emergence of governance norms in volunteer-driven FOSS communities, and what effect these norms have on the community as it grows from a new initiative to a large size organisation. A qualitative, embedded, multiple-case study of the inside view of social norms in three communities was performed. It may be considered a mixed method study design that combines the multiple-case study with theoretical modelling based on personal observation and experience, however that personal experience is also embedded within the same cases. ${ }^{33}$

The cases analysed in this study are large, mature, successful volunteer driven FOSS communities. There are only a small number of communities that achieved this level of success over an extended period of time - probably about a dozen. Another key criterion for selecting the case studies was access to individual key community actors and the organisations' decision-making bodies. The author has access to internal information of some communities because of his own history as a long-term contributor. As there is only a small number of communities that reach the late growth stage, and these communities develop a strong cohesion and a distinct insider culture, an analysis from outside these organisations could not lead achieve the same level of understanding of how the communities function. The qualitative study design explains the interpretive, experience based, situational character of the cases, and facilitates analysis of organisational development as a long-running, episodic and evolving phenomenon. A small number of community cases were chosen to avoid stereotypical generalisation caused by an unwarranted higher level of aggregation. ${ }^{34}$ The decision in favour of a qualitative research approach was supported by the assumption that quantitative methods do not promise reliable insights given such small constituencies. Experiments also were not considered feasible.

The study was conducted by performing 16 interviews with long-standing contributors to the communities who were either founders or who rose to community leadership positions at a later stage. Some of them are still active in these communities today. Some have resigned from their functions. Overall, the interviewees who contributed to this study represent more than 200 personyears of FOSS community leadership experience. The interviews gathered information about the personal ethics and convictions of the contributors and their interpretation of how the community governance norms and organisational design have developed.

The interview concept was developed against the theoretical framework (see section 2) which was built upon the individual experience of the author and the current state of FOSS community

32 Eric S. Raymond. The Cathedral and the Bazaar: Musings on Linux and Open Source by an Accidental Revolutionary (O'Reilly Linux). O’Reilly, Oct. 1999.

33 Robert K. Yin. Case Study Research: Design and Methods. 3rd. New York: SAGE Publications, Inc, Dec. 2003.

34 Robert E. Stake. Qualitative Research: Studying How Things Work. 1st ed. New York: The Guilford Press, Mar. 2010. 
governance research. The interviews provided evidence that puts the experiential expectations into perspective.

The interviews helped obtain unique interpretations held by the interviewees, to aggregate information from many interviews, and to access the personal experience of the interviewees that would otherwise be unobservable. The interviewees provided data about their own individual positions and their interpretation of questions about the community as a whole. By connecting observational insights with understanding of the individual ethics and convictions and observations on community governance by the interviewees, expectations in the theoretical framework about community governance norms and contributors expectations can be tested. ${ }^{35}$ The final report is based on personal observation, the interview results and information available in artefacts like minutes from general meetings, statutes, manifests or codes of conduct that the communities published.

The qualitative method chosen leads to limitations in the applicability of the study's results. The emphasis on the inside view regarding governance norms does not take external factors like the explosive growth of FOSS into account or considered the fact that this may also have contributed to community growth and other development trends.

Demographical changes affect communities - one interviewee mentioned a perceived decline in the proclivity of individuals to volunteer for social causes. Market trends that affect the position of the community's products also probably play a role. More importantly, the subjective, personal, constructivist point of view applied in the study means that observations only represent the experience or interpretation of the participants and the author, not necessarily a true meaning. The findings in this report can therefore not be generalised. They should however provide a valuable deep understanding of the inside view the communities and contributors have on themselves.

\section{The mindset behind community governance}

The interviews for this study consisted of three separate parts. The first one focused on the individual contributors, what their expectations and convictions were and why they joined their communities, how these expectations developed or changed over time, and what principles or ethics of individual conduct are important to them. The second part of the interview focused on the community as a whole, and the third part discussed inner-community conflicts as focal points for governance debates.

This section is based on the first part of the interviews. Some of the key governance documents like organisational statutes, the codes of conduct or community manifests have been authored by the interviewees. It is assumed that since the interviewees are founders or long-time participants in the communities and through their leadership roles actively influenced the community constitutions, their expectations and convictions strongly influenced the emerging governance norms. Even if these may have changed at a later point in time, their influence should still be apparent.

\subsection{Engage in a community of makers}

It is commonly assumed that participation in the development of FOSS products is primarily needdriven. ${ }^{36}$ However, the need for a solution to a particular problem does not explain sustained investment of effort into being a community member in good standing. To justify this behaviour, being part of a community requires that additional rewards like a sense of belonging are generated. The most limiting factor to contributors is the time available to undertake such intrinsically

\footnotetext{
35 Ibid,

36 Eric S. Raymond. The Cathedral and the Bazaar: Musings on Linux and Open Source by an Accidental Revolutionary (O’Reilly Linux). O’Reilly, Oct. 1999.
} 
motivated work. The different projects compete for this time from individuals. ${ }^{37}$ Being an integral member of a community means sharing time available between creating contributions to the product, which is perceived as a fun, productive and creative activity, and progressing through a social hierarchy involving engaging in community processes, which may be considered a necessary but time-consuming overhead. The initial question to ask is why contributors that end up being involved over an extended period of time and investing significant amounts of their personal time into FOSS contributions make the decision to join the community in the first place.

All participants in the study stated that the impetus to engage with the community and become a part of it was to contribute to the community's main product. They also stated that the creation of that product needed to be a positive challenge to motivative them and not be a routine task. Only a third of interviewees initially chose a specific community because of its social norms. A strong majority however said that over time, being a part of the social group became more and more important to them. A common phrase used to describe this phenomenon is "come for the technology, stay for the people."

The freedom to choose what task to work on in a group of like-minded people and the creativity in the search for a solution that this affords was mentioned by almost all interviewees as a motivator for becoming part of the community. This indicates that contributors are attracted to a FOSS community because of the challenging products it sets out to create, and only then learn about the social norms within the community and begin feeling attached to them. Those interviewees who are not active in their communities anymore usually exited gradually, reducing the amount of their contributions over time until they stopped. The expectations on an individual's productivity are in line with existing research which identified the sense of personal creativity felt by the contributor as the biggest impact on contributed hours. ${ }^{38}$

Multiple interviewees mentioned that they felt the community mission was "worth fighting for" in that it combines a productive, creative activity with a sense of contributing to a greater good, like fostering technical innovation, building up competition to dominant proprietary products or advocating for the societal benefits of software freedom. The communities provide a virtual place where individuals who share this combination of rather specialised concrete need and ethical conviction congregate. Whilst this may readily exist online, it may not occur in a physical location which is of course less likely to reach a critical mass and become a gathering place for like-minded people.

FOSS communities are meritocratic in the sense that individuals gain influence solely based on their contributions to the combination of community product and social process. This environment attracts highly skilled individuals who interviewees felt they could look up to and learn from, but at the same time who accepted them as equals. Such learning is a rare opportunity not commonly available to highly skilled individuals in physical environments. Meritocratic peer status based on concrete contributions also leads to a notable absence of other forms of discrimination by for example nationality, race, gender, age or other factors, at least initially. Individuals with non-binary sexual orientation are a common sight at community events, and do not usually attract much attention. One interviewee assumed a higher-than-normal share of individuals with symptoms of autism or Asperger syndrome amongst the contributors.

\subsection{Equality of opportunity among peers}

The interviewees joined their communities when they were still in the initial or medium stages. Some explained the perceived group size as "tiny" or mentioned that there was a positively motivating

37 Karim Lakhani and Robert G. Wolf. "Why Hackers Do What They Do: Un- derstanding Motivation and Effort in Free/Open Source Software Projects”. In: Social Science Research Network Working Paper Series (Sept. 2003). 38 Ibid. 
"David-vs-Goliath" feeling to working towards the group's goals. Most explicit and implicit governance norms were established in these phases. If communities needed to provide a combination of productive contribution opportunities and matching ethical convictions, what were the expectations of the contributors when they joined in respect of how these communities should operate?

Only a minority of the interviewees joined their communities with expectations of their governance norms. Some of the community processes in fact came as a surprise to newcomers, for example the extent to which new participants were immediately accepted into the group and even encouraged to represent the project at community and other events. Some intentionally joined the community to learn about how it works and stayed in an observer role for a period of time. Most participants developed their preferences towards governance norms while being a contributor.

Most of the interviewees emphasised a priority of "doing" over "talking". Contributors to the KDE community are very conscious of the "who does the work decides" rule. While an absence of discrimination is generally expected, the meritocratic rule within the communities does not translate to egalitarianism. Participants earn their prestige or even the right to participate in debates within the community through the contributions they make. This translates to an expectation of equality of opportunity, but not of an equality of rights. Ideally, the status any contributor might obtain depends on how much effort she or he invests into contributing to the community's causes. Community members who "only talk" found little acceptance and were sometimes explicitly denied a voice in debates. Some stated that initially they felt like the community needed only the grass-roots meritocracy structure, but that in later stages they changed their mind about that.

Almost all interviewees mentioned an inherent tendency to form sub-groups within communities specialising in particular functions or product aspects. These sub-groups remained at a size that continued to allow for ad-hoc coordination, even as the overall community grew beyond a size where this would be effective. The governance within these sub-groups was less standardised, one interviewee described them as "little villages with chieftains". Sub-groups also helped to retain regional or cultural cohesion and the sense of productivity by isolating their members from what some described as excessive debate. Because they initially associated themselves with one of the subgroups, the communities felt smaller to the interviewees at the time they joined than they really were in numbers of overall participants.

Surprisingly, the fact that the community product is distributed under a free software license or generally is a common good was not mentioned as an expectation by the interviewees, but as a precondition. Similarly, the absence of discrimination is expected as a given. Some said they would simply not consider participating in any initiative unless the outcome is freely available to all.

\subsection{Balance of makers and community builders}

The communities in this study all succeeded in establishing themselves as important in their respective fields and grew from the initial stage to the medium stage within two to four years. Almost all interviewees mentioned that being a member of the community became a goal in itself. Where previously, community membership was a means to facilitate contributions to a product, the contributors built personal attachments to the community as a sort of virtual home, where they maintained friendships and developed loyalty to the group. Some of the early contributors quickly rose to community leadership positions that became an important part of their self-perceived identity. They reallocated a share or all of their available time to community management tasks, reducing their product contributions in the process. Differentiation emerges between the product developers as the makers and the community builders as the maintainers.

Most participants could not rely on previous experience in managing larger communities and were 
surprised by their own success. One interviewee involved in Wikimedia explained how the explosive growth of the community in 2004 caused the group to consider how to coordinate "once we were more than three". The communities struggled with the transition from the initial stage. For multiple interviewees this transition happened when they realised that they did not know all contributors personally anymore, which also indicates a breakdown of ad-hoc coordination. The KDE core team retreated into private invite-only mailing lists where "those who do the work" could coordinate. The need for organisation and administration manifested itself when the community started to organise the first all-hands conferences.

To manage funds and donations the legal entity of KDE e.V. was created. The reliance of private communication channels was felt to contradict the expectation of transparency and open process by one interviewee. However, it was considered necessary at the time, and is still in place today.

Contributors that mainly work on community building face a dilemma that only becomes apparent over time: while they are contributing to core functions of community management by being board members or project representatives, they are not taking part in product development anymore in a community that primarily exists for that purpose. Consequently, their merit eventually declines. Some cling to their influential positions, possibly realising that they will not be able to maintain their community status once they hand over to a successor. Progressively they disconnect from the product-focused elements of the community and begin to value procedural questions about community management higher than facilitating the productive processes. Instead of being supportive, the administrative entities created by the communities exhibit a tendency to grow to be antagonists to the community of makers, "with members who contribute little and a board that contributes even less", as one interviewee described it.

\subsection{An ambitious, productive meritocracy of equals}

Over the years that the participants in the study contributed to FOSS, it can be assumed that it is common for them, once in a while, consciously or sub-consciously, to take a step back and to reconsider whether their time and money spent for the community's purposes is still a worthwhile investment. We asked them what criteria they apply when evaluating the perceived quality of their community. The answers were surprisingly uniform across the participants from all three communities.

All or almost all interviewees agreed to the following criteria:

The communities need to provide a welcoming, inviting culture. It forms the basis for the close social connection that develops between contributors. The communities should also extend trust to newcomers, allowing them to learn the community norms even if it involves making mistakes. This includes flat hierarchies for contributing to the community products. The communities implement an "open door policy", as KDE puts it, where newcomers, once they have an account, have access to almost all of the project's infrastructure. Common well-accepted exceptions are system administration, legal and financial functions.

Participants expect their communities to implement meritocracy. While the understanding of meritocracy is not completely uniform, regarding what constitutes a contribution and how it should be valued, the prestige and influence of contributors within the community should be measured by the aggregated value of their contributions, and nothing else. Two aspects of meritocracy are less defined in this regard, how merit diminishes over time (forcing old-timers to make way for new contributors), and which other soft factors like socialisation, being in the right place at the right time, gender or age, influence merit. More recently, liberal contribution policies as applied by the Node.js project address these issues. ${ }^{39}$ Experienced contributors consider meritocracy in FOSS communities

39 Mikael Rogers. Growing a contributor base in modern open source. 2016. url: https://opensource.com/life/16/5/growing- 
very important but question the naïve understanding of meritocracy that is commonly applied.

In this respect, there is an expectation of equality of opportunity. All communities, even if they down-play it, form status groups like administrators, formal members of the organisations or elected positions, which have noticeable barriers to entry. A common expectation is that all these positions should be open to anybody willing to contribute enough to the community cause, subject to a common set of criteria. Equality of opportunity is different from meritocracy in that status and merit may diverge. Individuals who attained status based on past merit that has now diminished may still be influential in the organisation. Similarly, a valuable contributor may have merit but not advance in status because, for example, no elected positions are available at that time, or old-timers get elected to them. This may cause personal disappointment, possibly disengage valuable contributors and eventually cause them to spread negativity or even leave the community.

Contributors are looking to contribute to useful, productive communities. They want their contributions to help the community to get closer to achieving its goals. It is often not enough to contribute to the product, contributors also expect the product overall to be useful, and to receive feedback or even to get more contributions from users outside the community. One of the main values that the community adds to the peer production process is to add distribution and communication channels to attract users to the community products and create a feedback cycle back to product development. ${ }^{40}$ An increase in the required share of available time being spent on community-internal debate detracts from the sense of productivity.

On top of the community helping them to be useful and productive, contributors expect the community's mission and vision to be ambitious. It is not enough in the long term to "build a better mouse trap", as the intention to build a FOSS replacement for a proprietary product has been described. Achieving societal change towards software freedom by lobbying for it is considered an ambitious goal, as is freeing a large user bases from lock-in to proprietary products, or creating "a world in which every single human being can freely share in the sum of all knowledge", as in the Wikimedia vision. In an abstract sense, contributors want the community to aim at making the world a better place, and for their contributions to help with that.

Next to these four quality criteria, the interviewees mentioned aspects that pose preconditions for engaging with a community. These preconditions may be considered hygiene factors, criteria that do not positively motivate contributors, but whose absence would be considered a reason not to engage with the community at all. ${ }^{41}$ Such factors include that community products are public goods, an absence of discrimination, a positive communication culture or code of conduct, respect for minorities, reasonable escalation mechanisms, supportive technical infrastructure, and opportunities for learning and personal improvement. These factors are "basics that need to be there".

We asked the participants if they felt a sense of responsibility for or loyalty to the community as they progressed, which they unanimously agreed they did. Some felt that the team they worked with started depending on them, and even tried to empower their colleagues to reduce that dependency. It would have felt bad for them to leave the community while this dependency existed. The merit they attained and the personal relationships built with other community members gives them a sense of responsibility for the community as a whole. They also understood that it would be hard for them to replicate the time and effort invested, which imposes a cost on exit that makes it difficult for longterm contributors to leave the community. ${ }^{42}$

contributor-base-modern-open-source (visited on 03/13/2019).

40 Yochai Benkler. "Coase's Penguin, or, Linux and "The Nature of the Firm"'. In: The Yale Law Journal 112.3 (Dec. 2002), pp. 369+.

41 F. Herzberg, B. Mausner, and B. B. Snyderman. The Motivation to Work. Transaction Publishers, Jan. 1993.

42 A. O. Hirschman. Exit, Voice, and Loyalty: Responses to Decline in Firms, Organizations, and States. New edition. Harvard University Press, July 1970. 
The communities struggle with continuing to be inviting to newcomers. Both product- and processrelated barriers to newcomers emerge and grow over time. In the initial phase, all contributors are newcomers and mistakes are commonly seen as part of the process. Later, longer-term contributors have accumulated experience, and the quality gap between contributions by them and by newcomers widens. The German language Wikipedia for example introduced a pre-publication review ("Sichtungsprinzip"), which reduces the trust extended to new contributors. This reduces the feeling of appreciation and acceptance that the participants reported they themselves felt when they started contributing. Interviewees said that the trust that contributions are generally valuable has been lost to some older community members.

\subsection{Ethical principles applicable to community governance}

Contributors will only be intrinsically motivated to voluntarily spend significant efforts in a social group that conducts its activities in a way that agrees with the ethical convictions and principles of the individual. Such convictions are formed through life and rarely change. They can be considered an external variable that the governance norms of the community should reflect. We asked the interviewees which principles that are considered "just" in other social groups, they think, should also be applied in their communities.

The interviewees strongly agreed that working code, meritocracy, solidarity and transparency are key principles that they look for in the governance of their community. Working code refers to the expectation that "code should speak louder than words", meaning that concrete code or other contributions to the community product should be valued higher than "politics". This argument is related to a general paradigm that postulates that FOSS development should focus on delivering a working implementation over, for example, writing a detailed specification. ${ }^{43}$ They feel that the work on the product should be the benchmark by which the community is judged. This principle is important to contributors because it describes very directly how the communities should operate.

Meritocracy is mentioned again as an individual expectation, indicating that the term is not only used to describe a mechanism of community management, for example in codes of conduct, but also as an expectation of a norm that directly influences the motivation of individuals to contribute. Communities implement meritocracy because their contributors expect them to or would otherwise not participate.

Solidarity is a principle that shows itself in an extension of trust to newcomers and more experienced contributors, a belief in their generally good intentions, and a habit of mutual support. It is part of the fabric of the social cohesion that the communities form and enables them to overcome otherwise separating attributes like race, gender, nationality or age. Tensions in debates have often been resolved in good humour by invoking Hanlon's razor, reminding everybody involved not to attribute to malice what can be adequately explained by (collective) stupidity.

Transparency is a common expectation that should result in processes and debates that are accessible equally to and documented for all contributors. This is understood as an invitation to participate, not a duty. The transparency principle is to a large extent engrained in the technical infrastructure of projects. Discussions take place on mailing lists, wikis or online chats, and are commonly logged or otherwise preserved. Activities are coordinated in project management tools or task trackers, often in ways similar to how a software development project would be organised. This habit may be encouraged by familiarity with software engineering tools. Interviewees from all three communities mentioned that they feel like their organisation is not as transparent as it should be with regard to governance processes, as opposed to product contributions.

There is no agreement on whether or not communities eventually need to fall back to majority

43 Steven Weber. The Success of Open Source. Harvard University Press, Apr. 2004.

Journal of Open Law, Technology, \& Society

Vol. 9, Issue 1 
decisions. Some interviewees believe that if the community cannot reach consensus on a subject, it is better if no decision is made at all. Others accept that situations exist where making a decision is inevitable. When asked directly, most but not all would prefer a decision over non-decision. All understand that with voluntary participation decisions cannot force contributors to act in a certain way. However, there was also disagreement over whether or not consensus should be sought as the decision-making principle. There is little awareness that, for many issues, staying with the status quo is one of the alternatives to choose from, and that not making a decision is equivalent to deciding to stick with the status quo. The consequences of decisions are evaluated, but those of indecision are commonly not.

The expectations regarding decision making processes appear to change over time, with multiple interviewees reporting that during their early involvement their preferences have leaned much more towards unstructured ad-hoc coordination, while after being involved for a number of years they feel that better defined and documented decision-making processes and especially escalation and conflict resolution mechanisms become necessary. It is not clear if this change is caused by gaining more personal experience, or by the communities outgrowing the initial and medium stages and operating as larger organisations.

The concept of transparency is connected to a number of common expectations regarding what constitutes a FOSS development process. Habits like open technical discussions, online collaboration and releasing working code early and regularly are considered strengths of the wider Open Source community. To enable that, a contributor or newcomer should be able to understand what the community is working on and how to take part in it based on information available online. Contributors also need to ensure that they possess all rights to use, study, modify and distribute the community product without a need for later negotiation. The emphasis on transparency is born out of the necessity to facilitate distributed collaboration in a diverse team.

There is an understanding that the communities implement these principles well with in the production processes, but not so well in community governance. In particular, a lack of transparency and meritocracy is noticeable in the decision making of the community leadership and in staffing high ranking community functions. In terms of documented structures and processes, the communities do not differentiate between product and governance related decisions, despite the fact that many of the norms applied rely on the fact that technical changes can easily be reverted.

The interviewees mentioned that there is a close match between their personal ethics and convictions and the social norms they expect the communities to develop. The fact that the governance of the communities is modelled so closely after their ideal of how an organisation that benefits the common good should operate is a strong motivator for them to continue contributing.

\section{Case studies}

The communities studied for this report are primarily volunteer driven (their contributors are amateurs in that their community engagement does not constitute a significant direct source of income, as opposed to professionals), mature (they have been working towards their purpose for multiple years), comparatively large (they have attracted between dozens and hundreds of contributors over time) and successful (each of them is recognised as an influential organisation in their respective field). Even though all of them produce freely-licensed public goods, the communities differ in the nature of their main product: the KDE community primarily produces software with a focus on end-user needs, FSFE is a free software pressure group that advocates the benefits of software freedom and Wikipedia produces an online encyclopaedia. The community product is the key element that provides participants with the opportunity to contribute. However, the communities have been selected using the hypothesis that community composition has a more 
dominant impact on governance norms as they emerge than the nature of the main community product being created.

Governance norms are expected to develop according to the expectations and convictions of the contributors in respect of the characteristics of the collaborative peer production process.

The following section analyses the vision and mission, the formal and informal organisational structure, the decision making and conflict resolution mechanisms and the rules for group membership of each of the studied communities in each community.

\subsection{FSFE}

\subsubsection{Mission, foundation and history}

FSFE was founded in 2001 with the mission of bringing about sustainable change towards societal freedom in the use of digital technologies. The ambitious "life-time scale" ${ }^{44}$ of this mission was understood as comparable to initiating "a second enlightenment" with regard to software freedom in Europe. At the time of foundation, FSFE was considered the European sister organisation to the FSF. The organisation gained recognition by representing the wider Open Source community in the anti-trust case against Microsoft's dominant position as a supplier of operating systems for personal computers in the European Commission. FSFE also represented the community at the World Intellectual Property Organisation and the Internet Governance Forum.

FSFE introduced a fiduciary licensing program in 2003 that allows FOSS contributors to have their copyright ownership managed by the organisation. The FLA program strengthened the role of FSFE as a representative organisation of the European free software community.

In 2005, FSFE launched its "fellowship" program, widening the base of supporters to those who wished to contribute to the organisation's purpose financially, instead of or as well as by investing personal time. The fellowship had a limited representation in the general assembly through two seats for finance contributing fellowship representatives, until 2018, when the fellowship program was terminated.

With the increasing adoption of FOSS in commercial products, the complexity of compliance with the free software copyright licenses and the danger of free-riding behaviour of some manufacturers became apparent. With support from external parties and in cooperation with gpl-violations.org, FSFE launched the Freedom Task Force, an initiative intended to help contributors and businesses to create and use software distributed under FOSS licenses correctly. The European Legal Network was founded in 2008 as a venue for legal and technical experts to collaborate on legal and licensing issues related to free software and quickly expanded beyond Europe through the support of key lawyers in the European community. The Legal Network is currently the single largest network of free software legal experts world-wide.

FSFE continues to grow in influence and size. Today, it wields relevant political influence at the European and EU member state level, has strong backing from the FOSS community, and hosts the most influential legal and licensing discussions globally. It employs a president as well as a small group of policy analysts, campaigners and administrative staff.

FSFE offers opportunities for FOSS activists to participate in a small set of well-defined key products - political influence on the regulatory framework relevant for free software, coordination of various regional free software related activities, and facilitating the discussion and promotion of free software

44 Quotes in this section are taken from the interviews, unless otherwise noted. 
legal and licensing topics.

\subsubsection{Formal and informal organisational structure and conventions}

The original intention of FSFE's organisational structure was a federated system of regional chapters with a central coordinating office that represented the organisation at the European and global level. Based on subsidiarity, the local chapters would be autonomous except when central coordination is needed. The concept of local chapters did however not materialise, with only one ever being active. The idea of local chapters was eventually dropped in 2016, leaving the organisation with a headquarters in Berlin that represents FSFE across Europe.

The legal entity of FSFE is an "eingetragener Verein", a charitable association registered in Germany. The statutes are the most explicit documentation of FSFE's structure and processes. The formal members of the organisation are somewhat misleadingly called the general assembly, even though it is a permanent decision-making organ. The general assembly elects the president and vice president. Historically, the president has been the most visible and influential role in the organisation. The president, vice president and treasurer together form the executive council, the de-facto day-today decision-making body of the organisation. Activities are coordinated between the FSFE team, the group that comprises all active contributors with separate communication channels and a formalised, email-based decision-making process. The general assembly and the staff are subsets of the team. Occasionally, task groups are set up to handle specific topics.

Informally, especially in the initial stage of FSFE, some individuals exercised significant influence without a formal mandate and were called "luminaries" by some interviewees. Since FSFE was founded as a sister organisation with the "blessing" of FSF, early activities where coordinated with FSF, and approval was sought for key political positions and messaging. The influence of FSF waned over time, also because FSFE applied a more collaborative style of governance than FSF. Approval of general assembly membership is handled very selectively. There are no term limits or requirements of re-election for general assembly members. Early-stage participants still wield significant influence in the general assembly, even though some would not be considered part of the team today since they are not actively contributing. The approval for full membership in the organisation is selective and depends on a combination of individual initiative and pull from existing members. As of May 2017, there have been 27 full members, with about two thirds actively contributing in the past 6 months.

To some interviewees, the formal organisational structure no longer reflects reality. They consider the loosely defined team as the core of the organisation, since most of the day-to-day work is coordinated amongst them today. The team however does not have authority over budgetary or executive decisions that are a prerogative of the general assembly, marking a significant deviation between power and responsibility. Many conventions are implicitly defined and passed on by word of mouth. Long-standing rules may still be in effect but are not very well known or followed. Some norms and processes are clearly under-documented, which one of the FSFE founders during the interviews classified as a "rookie mistake".

Due to its history and initial community composition, FSFE has a regional concentration in Switzerland and Germany, with the head office being located in Berlin. Since almost all activities are conducted online, the impact of the community is spread relatively equally across Europe. Local (country) teams exist in 8 European countries as of January 2019 and, in the case of the European Legal Network, globally. ${ }^{45}$

45 https://fsfe.org/about/localteams.en.html

Journal of Open Law, Technology, \& Society

Vol. 9, Issue 1 


\subsubsection{Decision making and conflict resolution}

Most decisions are made at the FSFE team level in a consensus-driven, mainly email-based process. An issue is raised on the team mailing list. After deliberation a proposal is submitted to a specific decision mailing list. The proposal is accepted if no rejections are raised. In the case of objections, the proposal is returned to discussion, refined and re-submitted in an updated form. While this process could theoretically repeat multiple times, the consensus-driven culture within the group limits iterations so that almost no proposal reaches a third round of debate. If the team realises that consensus cannot be achieved, the proposal falls back to the president who then may abandon the proposal or, if considered necessary, force a decision. There is awareness of the need to find resolutions that are palatable to those who raised objections. The value of the decision is weighted against the cost of demotivating members of the team.

The general assembly decides based on a simple majority after detailed deliberation of an issue. Only a few more strategic decisions are made at the general assembly level. The local groups develop their own processes that are not prescribed by the central office. This approach works well since those groups are small in size.

In general, there is no defined way to appeal against a decision. Staff are supposed to direct complaints against decisions to the executive council, in which the president is one of three members. There is no process to appeal against general assembly decisions.

Overall, these convoluted and circular rules of appeal potentially result in an absence of accountability across the organisation. This is balanced by the dominant motivation to work towards a common goal, however there are no protections against abuse. Compliance with norms and processes is effectively left to chance.

The decision-making process within the team and guidelines as to how the general assembly and the organisation as a whole should work were documented early on in FSFE. One of the interviewees assumed however that only a small fraction of those currently active in the organisation are fully aware of them. While staff and general assembly members may assume they are commonly understood, these documented norms and processes are not transparent to anyone outside the general assembly and organisation's staff, and so create a barrier to newcomers' effective participation . As a result, reforming the formal structure has proven to be very difficult.

\subsubsection{Community membership, roles and privileges}

Throughout its initial stages when FSFE represented the free software community in anti- trust cases a significant risk of elitism was felt by participants. Formal membership in the organisation was dependant on approval of the existing members and applied selectively. This risk continues to be perceived as relevant by some in relation to structural reform today. There are significant barriers to entry and a selective approval process to formal membership in FSFE.

The vast majority of FSFE contributors are not formal members of the organisation. Governance is indirectly affected by this as the strong influence of long-term contributors or staff as an unrepresentative membership can be used to influence which issues are put up for a community decision-making. Multiple attempts at organisational reform in recent years ended in indecision.

There is an ongoing argument as to the extent that the formal structure should influence the work of the community. While contributions to FSFE's mission do not require formal status in the organisation, the lack of clarity regarding ways to participate and to gain access to key roles may have a detrimental effect on contributor engagement. Interviewees pointed out that contributors commonly slowly fade away instead of leaving with a clear end to their engagement. This makes it 
difficult to measure levels of engagement, like the number of active contributors or contributions made in a time frame. The effect of the social structure on the success of the communities is not explicit.

\subsubsection{Structural reforms and outlook}

Similar to the other case studies, the formal organisation of FSFE has rarely changed. The fellowship program was introduced in 2005. The fellowship seats in the general assembly combined with the fellowship representative elections existed from 2009 to 2018, as did the role of executive director. There have been no other major governance changes to the organisation since 2009.

A strategy process was started in 2013 and is still ongoing. It was designed as a top-down process and mainly involves the inner circle of general assembly members and staff. The wider Open Source community, particularly that outside of the regional focus area of German-speaking countries, is not involved. While the process produced statements of intent, it did not influence the day-to-day work of the community much or trigger an alignment of activities across the subgroups. This situation indicates a lack of forum for strategic discussions where all stakeholders engage, such as an actual "annual general assembly" across a wider membership. The issue is exacerbated by some members' position that FSFE is not accountable to the wider Open Source community and only speaks for its members. An interviewee summarised the strategy process as "a lot of discussion and very little results".

According to the interviewees, there is no systematic process to maintain and document the formal structure or to align it to the development of the informal one. Some rules are being ignored since the problem they anticipated, like a hostile takeover, has not occurred. To newcomers the governance of FSFE is hard to understand and not transparent. Contributors that are not part of the staff or the general assembly have practically no chance of influencing the organisation.

An interviewee mentioned a perceived lack of impetus for change since about 2011, with FSFE's leadership mainly taking on a maintainer role (see section 3.3). This is exacerbated by a difficulty in creating effective collaboration between staff and volunteers. There is a chance that the contributions of hired staff displace volunteer work by reducing intrinsic motivation. This may create a potential zero-sum scenario where spending on personnel changes whether FSFE receives contributions from staff versus volunteers but does not necessarily change the overall level of contributions.

In summary, the formal and informal organisation of FSFE as well as its decision-making processes appear to have been well thought out originally but have not been updated in pace with the growth of the community and outside changes. The well-thought out organisation design has aged and is now outdated and in need of reform. It appears that the main problem is not with the quality of the initial setup, but with the absence of constant, gradual improvements to it over time.

The KDE community took a different approach, but due to a comparable lack of a systematic improvement process, ended up in a similar situation.

\subsection{The KDE community}

The KDE project was founded in 1996 by Matthias Ettrich when he wrote to the de.comp.os.linux.misc Usenet group looking for contributors to a new, visually pleasing, easy to use graphical user environment for the increasingly popular Unix operating systems, named KDE. Unix was regarded as the superior operating system to Windows, but the usability of modern graphical desktop environments on Unix systems was a real, widely felt limitation at the time. The call for contributors fell on open ears in software development circles. There was a concrete desire in the early contributors to build the kind of desktop they wanted to use themselves. The motivation of the 
founders and that of the initial stage community was identical. The possiblility of competing with the dominant proprietary desktops produced by large enterprises was an important motivation. Within less than a year, a small group of early contributors produced a first version of the new desktop that showcased the enormous potential for innovation in this niche.

KDE released multiple successful versions of its main product, the "K Desktop Environment" until the present day- version 1.0 in 1998, 2.0 in 2000, 3.0 in 2003 and $4.02008 .{ }^{46}$ By 2009, KDE had reached one million source code contributions, making it one of the largest FOSS projects at the time. $^{47}$

After the release of version 4.0, KDE changed the mission of its community to be an umbrella organisation that supports various free software initiatives, the desktop, now named "Plasma", being only one of them. In this move, KDE explicitly shifted towards emphasising the community process benefits over the development of the main product. Today, KDE is a large, still mainly volunteerdriven community with multiple regional subgroups (for example in Latin America and India). It still develops the desktop and other products and is networked and affiliated with the Open Source Initiative, FSFE and other FOSS stakeholders.

\subsubsection{Formal and informal organisational structure and conventions}

In November 1997 KDE e.V. was registered with the mission of representing the budding KDE community in legal and financial matters. It gained charitable status in 2012. The bylaws of KDE e.V. were the first and for a long time the only written constitutional document of the KDE community. They were also for the large part copy-pasted from unknown sources and not tailored to the needs of community collaboration. When the growing numbers of contributors led to difficulties in coordination, the core contributors, instead of building an overall structure supportive for the coordination of a larger group, retired into more specialised communication channels. Almost all other behavioural norms at the time have been implicitly assumed. In August 2003, an updated version of the bylaws drafted specifically to support the work of the community was approved. It introduced the concept of passive membership that enabled long-term contributors to remain members of the organisation when their level of involvement declined, without endangering voting quora, and codified the invite-only acceptance criteria for individual membership. ${ }^{48}$

Membership in KDE e.V. is reserved for active contributors to the project, who need to be invited to membership in the organisation by two existing members, emphasising a strong focus on individual contribution. Companies and other legal organisations cannot become full members, only (financially) supporting members without the right to vote in the general assembly. Employees of businesses may become members, but only on their own merit and in their own right. As a result of this the community is almost exclusively driven by volunteer contributors, with businesses invited to advisory roles. ${ }^{49}$ In August 2008, the KDE approved a code of conduct as the first documented community behavioural guideline next to the bylaws. In October 2012, the community published the "KDE Manifesto", which postulated norms like open governance, inclusivity and common ownership..$^{50}$

The KDE community operates in a decentralised fashion with KDE e.V. as a central support organisation and has offices in Berlin. Multiple regional sub-communities exist at different levels of formalisation. Some like the ones in Spain, India and Latin America are represented by individual legal entities that are associated with, but not controlled by KDE e.V.

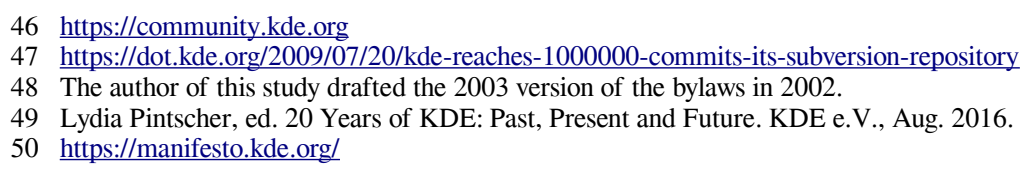


From the beginning, KDE e.V. was meant to support and represent the community. It was clarified in the 2002 general assembly that this excluded KDE e.V. from influencing the technical direction of the community product. The organisation is represented by the board and conducts an annual general meeting. In 2005, an attempt was made to establish formally recognised working groups that would coordinate with the board and be able to manage specialised budgets. Multiple working groups were established, but most ceased activities within a few years. No other formal structure has been defined within KDE e.V. ${ }^{51}$

From the start and in the initial stage, the KDE community considered itself a meritocracy. One of the first principles the initial group of about 10 contributors established was "(s)he who does the work decides", which postulates that even a community decision cannot force a technical direction on the person implementing it. The internal understanding was influenced by the publication of "The Cathedral and the Bazaar" 52 , which many of the early contributors had read. The group choose a meritocratic, egalitarian approach to self-organisation, with the original founder acquiring the most impact. Communication differentiated into different channels, particularly mailing lists, to keep the distraction of ongoing debate away from contributors working on the product itself. There was general acceptance of the argument that an inner circle needs to exist to manage the project, first with the kde-private mailing list and to this day with the non-public KDE e.V. membership mailing list. Access to this inner circle is granted by the existing insiders.

Informal behavioural norms played a significant role within the KDE community. There is a strong resistance to any form of authority within the community that is not based on individual merit. The rule that KDE e.V. shall represent the project, but not influence technical direction is considered a fundamental constitutional principle that newcomers are introduced to very early on. The resistance to authority was also embodied in the "(s)he who does the work decides" norm. As a result, technical direction developed organically from the activities of the contributors. Voting and other forms of formal decision making are not highly appreciated and seen as measures of last resort. Votes are commonly conducted to accept new members into the organisation, and to elect board members and representatives to external committees or organisations. The importance of these principles contributed to the absence of organisational design in the late community stage.

One of the key debates that has never been concluded is whether or not KDE e.V. represents "the heart of the community" or is meant to be a body that complements the community without being a core part of it. The strategy of the organisation was from the beginning that active contributors should be members of the organisation, and therefore jointly own and manage funding and ownership of trademarks and other assets. To achieve this goal requires an organisation that is accepted by a meritocracy, which needs to aggregate the interests of many of the core contributors.

However, the more influential the organisation became, the more it came to represent the project overall, with the board growing into a sort of project leadership. This created a conflict due to the fundamental resistance to authority prevalent in the community. The community had not established processes capable of making decisions on questions of this constitutional nature. Decision making relied on the relevant stakeholders taking part in an extended elaboration with the goal of reaching consensus. For technical decisions, this approach served the community well. The community does not differentiate between product related technical decisions and the implementation of norms of social process. The decision-making process aimed at consensus proved to be less efficient for topics that affected all community members, where everybody is a stakeholder. Effectively, the formal organisation became very difficult to change, with the consensus driven process affording each individual member a de-facto veto.

51 Diomidis Spinellis and Georgios Gousios. Beautiful Architecture: Leading Thinkers Reveal the Hidden Beauty in Software Design. O'Reilly Media, Inc., Jan. 2009.

52 Eric S. Raymond. The Cathedral and the Bazaar: Musings on Linux and Open Source by an Accidental Revolutionary (O'Reilly Linux). O’Reilly, Oct. 1999. 


\subsubsection{Decision making and conflict resolution}

Because of the "(s)he who does the work decides" rule, a decision "manifested itself based on what ended up in the revision control system". In the early and medium stages of community growth this approach served the community well. Possible differences would be settled by arguing for the cause until an agreement was found. The decision-making process relied on the organic coordination of a familiar, cohesive group with a common cultural understanding. Over the years, this attracted more contributors with the same traits, contributing to the common lack of diversity in FOSS communities: The contributors were predominantly young male software engineers. A growing community however requires increased specialisation and division of labour. Other community needs requiring skills like documentation, user experience design and community management were not met due to a lack of contributors as a result. Selective bias has been criticised as a hidden cost of meritocracies. ${ }^{53}$ It can be argued that the community may not reach the full contributor potential because of it.

Participation in debates is open to all contributors, but the impact of their voice depends on their meritocratic status. This is not unusual in smaller collective action groups. ${ }^{54}$ This attitude emphasises technical contributions over those in other fields. Minority opinions have a difficulty being heard. Because contributions are made voluntarily, there is only little participation of specialised and minority contributors.

Conflict resolution within the community is mainly absent. Except for appealing to the board of KDE e.V. as a general fallback option, there are no defined processes to escalate a conflict with the goal of settling it. Contributors are expected to sort things out amongst themselves. In 2008 the community working group was established, together with the code of conduct, with the aim to "maintain a friendly and welcoming KDE community, thereby ensuring KDE remains a great project enjoyed by all contributors and users". ${ }^{5}$

The community working group moderates using a participative approach and is not equipped with any sanctioning instruments. The only possible measure to sanction misbehaviour is a suspension of a contributor's accounts, either temporarily or, as an ultimate measure, permanently, a task performed by the system administration group. Account suspension constitutes a drastic measure, as it effectively removes the affected person from the community. It impacts contributors similarly to a citizen of a country being subjected to temporary exile or revoked citizenship or a church member being excommunicated. It also strips the person sanctioned from the means of communication needed to enable them to continue to be part of the discussion and defend their position.

Account suspensions are therefore issued only in a very few cases, and only after lengthy moderation failed to resolve the conflict. In some cases, this has delayed necessary responses to disruptive and abusive behaviour. Similar to the decision-making processes, the mainly informal conflict resolution mechanisms work sufficiently well in resolving product related debates with a small number of homogenous stakeholders with knowledge and a strong interest in the matter, and less well for issues relating to the community's social process with a large number of stakeholders with only moderate interest. This indicates that both the decision-making and the conflict resolution mechanisms were established in the initial and medium stages of community development and have not evolved to be suited to the late stage where the social process grows more important than the product aspects.

While this description of the decision making and conflict resolution mechanisms within the community may appear as criticism, it mainly aims to describe the observable results and developments. The KDE community had good reasons to establish these processes founded in the

53 Daniel Bell. “On meritocracy and equality”. In: National Affairs 29 (1972), pp. 29-68.

54 Olson Mancur. The Logic of Collective Action: Public Goods and the Theory of Groups. Revised. Harvard economic studies, v. 124. Harvard University Press, Jan. 1965.

55 https://ev.kde.org/workinggroups/cwg.php 
ethics of the community's early contributors. Well-defined formalised decision-making processes favour well-organised actors with the necessary resources to participate in these processes and who are expected to be businesses, not volunteer contributors. The absence of formal decision-making processes is seen as an emphasis of the role of the contributor of the product over others "that merely talk". Similarly, the apparent lack of conflict resolution mechanisms is also by design. One argument is that as long as the community has difficulties defining misbehaviour, it should not police it as that would result in arbitrariness. A second argument is that contributions that are disruptive to the overall technical direction of the community are important to innovation and should not be suppressed. ${ }^{56}$

The lack of formal definition of decision-making processes results in occasional over-the-top behaviour that consciously or subconsciously (even if with the intention of hearing all sides of an argument) prevent decisions from being made or from being implemented. Since there is no process that describes how discussions should be conducted, debates can become endless by bringing up a new arguments or points of view that need to be considered. It is not easy to distinguish between the contribution of an important argument to the debate and the deliberate raising of a tangential argument with the aim of derailing or prolonging it.

Bringing up tangential arguments that may or may not be critical to the conclusion happens often enough in the debates that it developed its own name, "bike-shedding",. This is named after the question of "which colour to paint the bike shed" when the debate is about "whether or not to build one". ${ }^{57}$ The result is that debates may take much longer than the subject warrants, a topic will not receive the necessary attention, a decision may never be made, or a decision once made will not be implemented.

Occasionally, the community applies "lazy consensus" where the debate is settled by a contributor committing a solution that reflects what has been discussed, pre-empting further discussion. This happens more often with matters where stakes are difficult to define or controversial. In the KDE community, the KOffice versus Calligra discussion ${ }^{58}$ or the decision to hire more staff, especially an executive director, are examples of discussions that dragged on for a very long time, sometimes years, before being concluded.

This pattern is even more difficult to manage because the individual contributor's reasons to prolong a debate may be sub-consciously self-serving but are rationalised towards the good of the community by the individual themselves, making them think they are acting in the best interest of the community. It gives individuals an instrument to abuse a participative debate culture that generally assumes good intentions. Instruments like time-boxing (limiting the period of debate by scheduling an executive decision or vote at the end) that are common in other collective action groups are not used in the KDE community because formal decisions through votes are not generally accepted. Interviewees suspected that the debate culture in the community was heavily influenced by the student lifestyle of the early contributors, dominated by non-structured arguments, a lack of any constitutional frame of reference and a lot of time for debate.

\subsubsection{Community membership, roles and privileges}

The KDE community implements an easily accessible "open door policy" to its core repositories, defined by the absence of any formal hurdles to gain access to the community infrastructure. Since all code and data is versioned, any change can be reverted, and there is no need for an approval process for contributor access. Only a small subset of the community infrastructure, for example the public-facing web sites, are kept under more restrictive control. Everybody who contributes to KDE

56 https://community.kde.org/Akademy/2013/ConflictResolution, there was no formal adoption of the suggestions.

57 Karl Fogel. Producing Open Source Software: How to Run a Successful Free Software Project. O'Reilly Media, Inc., Oct. 2005 .

58 https://lwn.net/Articles/419822/

Journal of Open Law, Technology, \& Society

Vol. 9, Issue 1 
products or the community ("everybody on the mailing list") is considered a community member. To participate in the product related aspect this is all a contributor needs.

In the very early stages, a private mailing list was created for the more involved contributors, with an invite-only membership policy. It later evolved into the communication channel for the KDE e.V. members that is still kept private. At the AGM in 2012, an attempt was made to change this communication to make it public. It ended in the creation of the kde-community mailing list, ${ }^{59}$ while the communication of the organisation is still private. There is privileged differentiation within the community regarding participation in the social aspect of community work, combined with significant barriers to entry like the invite-only principle. Contributors highly value being a community member, especially once becoming a part of the core team or KDE e.V.. Advancement to a role of formal community representative on the board or in external committees or foundations requires membership in KDE e.V. The KDE community is easily accessible for contributors to its product, but not as much to its social process.

\subsubsection{Structural reforms and outlook}

The formal organisation of the KDE community has been infrequently changed with the update of the bylaws, the introduction of the code of conduct, the publication of the manifest and the formation of the community working group, over the course of more than 20 years. Changes were incremental rather than disruptive, and retroactive in that they codified norms that the informal social process already had developed.

The informal organisation changed gradually but preserved "hacker culture". In the absence of formal structure, thought leaders have had a strong impact, with an emphasis on personality that is difficult to replace at a later stage.

If two contributors did not agree on which text editor KDE should ship, it would ship two text editors. There was no mechanism to influence technical decisions that affected the project as a whole and the users of the software. More importantly, there is no sanctioning mechanism to encourage activities that the community is interested in. The "(s)he who does the work decides" rule means that the user has to turn into a developer contributor to improve the software for her or his needs.

The community norms described have all been developed and adopted in the early stages of community growth. They worked well in the small to medium sized groups and did not change significantly in later stages when KDE decided to de- emphasise product development over being a community that creates FOSS products. An unresolved contradiction lies in the application of predominantly informal norms and ethics tailored towards a smaller coherent social group with uniform backgrounds and interests to the governance of a large, diverse organisation. The cultural foundation that the community codified in the vision and manifest is not implemented in its longstanding governance norms.

\subsection{Wikimedia}

\subsubsection{Mission, foundation and history}

The online encyclopedia Wikipedia was launched in 2001 with the vision of creating "a world in which every single human being can freely share in the sum of all knowledge". Unlike predecessor projects, it incorporated the idea that all content should be free with the same understanding as in free software. This vision was formulated in the early days of the project by the founder Jimmy Wales, and still remains largely unchanged.

59 https://mail.kde.org/mailman/listinfo/kde-community 
Wikipedia is created by the global Wikimedia community. There is no central authority within the community that manages activities globally. Instead, regional sub-communities operate mostly autonomously, usually along language boundaries. It is therefore difficult to describe the governance norms of Wikipedia as a whole. In terms of contributors, community activities and funding raised, Wikipedia has a strong presence in central Europe, especially the German speaking countries. This study focuses on the community of contributors to the German language Wikipedia and the supportive organisation Wikimedia Deutschland e.V. in Germany. Initially, Wikipedia was perceived more as an idea, a broadly collaborative effort to make the knowledge of the world accessible to everybody and to enable them to participate as a user, author or community member.

The idea quickly turned into a global movement that attracted large numbers of contributors. In May 2017, about 120,000 participants actively contributed to the project. With over five million content pages and nearly 900 million edits, Wikipedia has successfully built the encyclopaedia it set out to create, surpassing commercial encyclopaedias by article count and number of readers. It gained a large user base in the process, serving in average about 7.8 billion pages per month. It globally ranks 5 th in the list of most visited websites. With this initial success, some contributors shifted their focus towards building the very best encyclopaedia, with a focus towards quality over quantity. Others identified new fields and regions of knowledge that need to be captured and consider the task of collecting all relevant human knowledge far from completed. In any case, building the Wikipedia encyclopaedia is an ongoing, life-time scale undertaking. The question whether Wikipedia's mission makes it a project responsible for social change or for the concrete task or writing the best encyclopaedia in the world is still being discussed. ${ }^{60}$

\subsubsection{Formal and informal organisational structure and conventions}

There is only minimal formal organisation of the community of German-speaking Wikipedia authors. Wikimedia Deutschland e.V. represents the German language Wikipedia legally and provides community support. Similarly, the San Francisco based Wikimedia Foundation legally represents the global community and the English language Wikipedia and maintains a level of control over the regional organisations. These organisations however are not directly involved in coordinating or managing the work of Wikipedia authors and other individual contributors. Authors commonly focus on contributing knowledge in their own language and possibly to the English language Wikipedia, which is seen as the global fall-back. More than in other organisations, the contributor base is fluent, because it is possible to contribute, even anonymously, without much interaction with the organised community. Groups of regulars (Stammische, in German language) meet occasionally to maintain cohesion between the work of the individual authors. Many participants expect regular contributors to attend physical meeting to gain recognition. Editorial boards have formed for specific subject matters like chemistry or religion. ${ }^{61}$ Arbitration committees have been created in some countries (2007 in Germany) that assist in resolving conflicts between Wikipedia users. The arbitration committees do not interfere with regular contributor activity. ${ }^{62}$ Beyond that, no formal structure exists that the authors turn to for coordination of their work Intentionally, no attempts are made to unify the processes of the regional sub-communities. Regional differences and decentralised self-coordination are considered key strengths of Wikipedia.

Wikimedia Deutschland e.V. develops software used by Wikipedia, especially MediaWiki, lobbies for open knowledge politically, invests into free learning and free educational resources, provides infrastructure and facilities for use by volunteers, and overall manages the organisation's and the community's legal and financial footprint. In 2016, it reached 50,000 individual supporting members,

60 A history of Wikipedia is available at https://en.wikipedia.org/wiki/History of Wikipedia. Details about the vision can be found at https://wikimediafoundation.org/wiki/Vision. Content page count, number of active users and other metrics are available on Wikipedia's statistics page: https://en.wikipedia.org/wiki/Special:Statistics. A user is considered active if she or he performed an action in the last 30 days.

61 https://de.wikipedia.org/wiki/Wikipedia:Redaktionen

62 https://en.wikipedia.org/wiki/Arbitration Committee 
2,000 voting members, and about 85 employed staff. It is led by the executive committee (Präsidium) of up to 9 members, which appoints the executive director. The activities of Wikimedia Deutschland e.V. are for the most part considered orthogonal or supportive to the work of the community of authors. Some participants in the interviews actively refused the notion that Wikimedia Deutschland e.V. is part of the German language Wikipedia community and consider both separate entities. Wikimedia Deutschland e.V. does not consider itself responsible for the activities of the community of authors. The relation between Wikimedia Deutschland e.V. and Wikimedia Foundation has been characterised as that of "a far-removed sovereign" that tributes are paid to.

The community organisational structure has been described as "profoundly informal". In particular, early community contributors or "generally important top-dogs" can be very influential, even without formal roles. It was pointed out in the interviews that this may impose significant barriers of entry for new authors. Formal and informal structures have diverged significantly. It can be assumed that the community is not in a position to perform an analysis of the state of the project and derive conclusions for organisational reform, which has been classified as "negligent" in the interviews.

\subsubsection{Decision making and conflict resolution}

The decision making and conflict resolution norms within the author community show strong similarities to those found in the other studies, underlining the assumption that governance norms evolve based on community composition.

Acknowledging that majority decisions cannot be enforced against volunteer contributors; they have been replaced with non-binding opinion polls (Meinungsbilder). All active contributors may initiate and participate in an opinion poll. There are strong opinions about opinion polls, with some arguing that contributors should participate in them, and other arguing against participation. An aversion against formal decision making is obvious. Similarly, the "rule to ignore all rules" encourages participates to apply agency to their actions.

Intra-community conflicts are managed along a well-documented staged process from de-escalation to appeals to a mediation committee (Vermittlerausschuss) with volunteer members and finally to an arbitration board (Schiedsgericht) with members that are elected today by a qualified majority. Decisions of the arbitration board are considered binding within the community. Recommendations like remaining level-headed and assuming good intentions help to maintain a collaborative spirit, as do more formal guides like the "Wikiquette". While there are instances of "edit wars" or members acting under fake accounts ("sock puppets"), the conflict resolution process is mostly accepted and effective. These processes represent a mature understanding of the role of decision making, conflict resolution and of volunteer community dynamics. None of them involve Wikimedia Deutschland e.V..

\subsubsection{Community membership, roles and privileges}

Everybody who productively contributes to Wikipedia is considered a community member. Since anonymous contributions are allowed, contributors transition from loosely associated anonymous authors to registered authors known by a screen name and then may acquire additional roles like administrators. Elected Bürokraten (bureaucrats) manage administrator status. A number of additional roles exist that partially map to technical permissions in the operation of Wikipedia, like rolling back changes or inspecting contribution metadata. There is consensus that all contributors should be considered equals, taken seriously and valued based on merit. Even though being admin is foremost a technical task that allows to change other contributors' content, it is also implicitly a social role that needs backing by the community and therefore a strong standing or merit for the person acting as admin. Eligibility to vote is based on a minimum number of recent contributions, and since 
the bar is set rather low, has become a requirement for effective participation in discussions. Without it, an individual "would not be taken seriously". Long-term contributors who shifted their focus towards activities other than being authors sometimes struggle with that or produce edits to maintain their status. Social status within the community is closely related to contributions either of quality content or of the software used to run Wikipedia. Contributors to auxiliary functions like conference organisation or design are "not well known".

These status groups or roles represent contributor functions with a strong product focus - they are measured against their impact on the quality of the encyclopaedia. A remarkable disconnect was mentioned in the interviews between the legal entity Wikimedia Deutschland e.V. representing the German speaking Wikipedia, and the community of authors. One described the role of Wikimedia Deutschland e.V. as "collecting donations, being on TV, and attending galas, based on the work of the community". Multiple interviewees mentioned that being a member of Wikimedia Deutschland e.V. was perceived in the past as a negative factor with regard to contributor merit within the community and is now considered "acceptably eccentric". The role of Wikimedia Deutschland e.V. members within the community is largely irrelevant, except for a small number of contributors that try to participate in both organisations but find it time consuming and difficult. Wikimedia Deutschland e.V. has been repeatedly criticised for being disengaged from the community and not supporting it enough. Interviewees expressed that they believe the perceived under-performance of Wikimedia Deutschland e.V. is rooted in the lacking integration with the community, and that they "are happy if Wikimedia Deutschland e.V. is at least not breaking anything". Wikimedia Deutschland e.V. keeps authority of the budget and spends a significant share of the budget on nonproduct related activities.

\subsubsection{Structural reforms and outlook}

As with the other case studies, the formal organisation within the German Wikimedia author community has rarely changed. There is no structured process of organisational design review. In the past ten years, the adoption of the review principle, the arbitration court and the introduction of the visual editor are perceived as the major changes. Interviewees described the overall constitution of the community as rather "hostile to change".

Wikimedia Deutschland e.V. is aware of the rift between the author community and the formal organisation. It attempts to integrate the community through a collaborative planning and budgeting process and other activities. Decreasing author numbers create the necessity to act upon a perceived pent-up need for organisational reform, which is reflected in the annual plans for 2016, 2017 and 2018. Attracting and retaining volunteer contributors has been accepted as one of three key fields of action. However, less than ten percent of the overall revenue from donations and membership fees is allocated directly towards that goal. Wikimedia Deutschland e.V. positions itself as an organisation with the primary goal to foster Wikimedia projects. ${ }^{63}$ There is a profound feeling within the community of authors that Wikimedia Deutschland e.V. made itself independent and unaccountable. When asked what would need to change, one interviewee suggested that community members need to "get together" and re-take control of their project.

\section{Observations}

For the most part, the contributors agree on what they expect from their communities: They want to engage in a community of "makers". Amongst their peers, they wish to have equal opportunity to contribute. They understand the need for community management but want their communities to

63 "Wikimedia Deutschland [ist] im Hinblick auf die Wikimedia-Projekte daher als "Förderverein" zu verstehen." (https://meta.wikimedia.org/wiki/Wikimedia Deutschland/Präsidiumshandbuch) 
remain focused on being ambitious, productive meritocracies. They believe that there is strong solidarity between the members of their communities, and that "them-versus-us" conflicts between the communities and their leadership or the makers and the community builders are mainly absent. Still, the communities exhibit similar symptoms of distress: They have trouble growing their contributors and contribution count in a sustainable way, have difficulties implementing organisational change and get stuck making important decisions, resolving inner-community conflicts or enforcing the values of their social groups.

It is obvious that the contributors think highly of their communities. There have been no indications of any malicious intent by influential participants or abuse of the communities for their own advantage. Conflicts within late stage FOSS communities are more likely to reflect difficulties volunteer contributors have in collaboratively developing their organisations and maintaining control over their destiny as they grow to be large groups.

\subsection{Formal and informal organisational structure and conventions}

Formal organisation is not the first thing participants have in mind when starting a FOSS initiative. The groups are initially small and do not possess assets or liabilities that require an independent formal organisation. KDE and Wikimedia Deutschland e.V. added a legal entity that represents their community after their projects started. The founders of FSFE on the other hand were aware that their success depended on a strong, independent organisation, and started off with a carefully designed organisation that anticipated attempts of hostile take-overs and the creation of regional subsidiaries.

None of the three organisations implemented a systematic effort to periodically review and reform their formal organisation. Changes to the organisational structure have been very rare, resulting in a growing disconnect between the community's production processes and their governance related activities.

FSFE did not succeed in establishing thriving, decentralised, independent regional sub-organisations, and concentrated its activities at the Berlin head office.

The KDE community continuously restricted the mission of KDE e.V. to administrative support and resisted the delegation of authority to elected representatives. This led to long-standing contributors questioning its usefulness and contributed to an emerging culture of bike-shedding and indecision. The organisational structure of KDE e.V. was not changed even as the KDE community changed from a single product to an umbrella community. Eventually, KDE e.V.'s main role became to organise the annual KDE Akademy ${ }^{64}$ conference and to provide funding for contributor meetings. For a period of time, the KDE community became infamously known for its lack of coherence and decision making.

The biggest rift between product development and formal organisation seen in this study is exhibited by the German language Wikipedia community. Despite the minor differences FSFE and KDE have with their formal organisations, they are still seen as an integral part of the community. Some members of the community of Wikipedia authors however wish that Wikimedia Deutschland e.V. would "not interfere with their work". Perhaps because being a Wikipedia author is explicitly not considered a selection criterion for employment at Wikimedia Deutschland e.V. the community of volunteer authors and the formal organisation that bears the community's name have diverged.

The performance of the support organisations in this study is not linked to the efforts invested into the original organisational design. Instead, given the absence of a systematic review and reform process, the organisations' ability to serve their communities deteriorated as they went from the

64 https://akademy.kde.org 
initial to the medium to the late stage. The aversion of the contributors against authority and "bureaucracy" put the need for reform in question and reinforced this trend. It is not the initial design that counts, as the organisations need to continuously adapt and improve the performance at which they support their communities.

All three organisations rely heavily on informal organisational structure. There is strong agreement between the interviewees that the documented formal structure is not implemented in reality, and that today the organisational structure of the communities is mainly implicit, well-understood only by early community members, and not well-documented for newcomers. Only a few fully know and understand the existing formal rules as they stand today. The divergence of formal and informal organisation and the lack of supportive performance of the community organisations is not currently perceived as a relevant problem. The resulting effects, enforced by lack of positive competitive selection of community leadership or inhibited acquisition of new contributors, are detrimental to long-term community growth and success.

\subsection{Decision making and conflict resolution}

It is commonly part of the spirit of a FOSS community that decisions should be made by consensus, that authority and hierarchy should be avoided, and that there should be minimal to no policing of contributor activity. These are all positive, defining aspects that are important to contributors. But do they match reality when compared to the decision-making processes and conflict resolution mechanisms of late stage communities?

The results from the interviews strongly suggest that all three communities make use of very few defined decision making processes, do not routinely apply instruments for shaping debates, experience extended bike-shedding and indecision regarding issues that are considered important, and that influential individuals - often project founders or early contributors - wield soft and hard vetoes over community decisions.

Most day-to-day decisions are made at the level of subgroups that focus on particular aspects of the community product. In these relatively small groups, informal decision making still succeeds. It is possible to understand the likely outcomes of the decision, and there is a joint sense of responsibility for that result. There is also no need for an appeal mechanism. If the outcome of the decision is not what was expected, the group again jointly decides on a new course of action. These are the decisionmaking mechanisms the communities developed in the early stages and that served them well.

Late stage communities also need to make more complex decisions, like hiring an executive director, organising a global conference or redefining the overall community vision and mission. These may involve trade-offs of resource allocation between subgroups or competing goals. The community as a whole is a stakeholder in these decisions. The outcomes of the decisions may be harder to predict, and unlike most technical decisions difficult to reverse. Undefined and informal decision-making processes, a lack of routes of appeals, and an excessive debate culture that may prevent decisions from being made have a detrimental effect on contributor motivation and pose a significant barrier to entry into higher level community functions. Early contributors stay in community leadership roles too long, at the expense of later contributors not assuming leadership roles even if their merit within the community would warrant it. The auto-organised decision-making mechanism of the subgroups fails when applied to higher level large group decisions.

Authority is commonly assigned to specific community functions, like the president in the case of FSFE, or the board in KDE e.V. There are no checks and balances to decisions made by these functions. Even if it is known to some participants that a way to question a decision of the president is to submit an item to the agenda of the next general assembly, this is far from obvious to the wider community, and also not communicated. There is little understanding that for every community 
function with authority a check needs to be implemented to allow oversight and for decisions to be appealed. In volunteer-driven communities with self-referential authority, this means that unresolvable issues eventually will escalate to a community all-hands decision. In turn, this requires a mechanism for community votes. Communities have a well-founded aversion to majority decisions considering that all contributors participate in the communities voluntarily. However, as a mechanism of last resort, no better alternative has been presented. Every decision should be appealable. Many of the long-term contributors interviewed in this study today acknowledge the need for well-defined decision-making processes, even though it took a long time for them to reflect on and change their initial preference for auto-organisation at all levels.

With unclear authority, it is difficult to apply instruments to shape debates so that a decision can be achieved in a reasonable time frame. Discussion drag on "until nobody has any energy left to disagree". A "fulsome optimism regarding the decisiveness of a large group" can be observed. Discussions are kept alive by influential contributors to avoid their conclusion with an unwanted decision. Shaping debates does not necessarily require voting mechanisms. Time boxing by asking that a consensus be reached after a specified discussion period and announcing a formal vote otherwise is one option. Relying on rough consensus combined with a clear procedure of appeal is another. The KDE community had good experiences with a "debate manager", a contributor that voluntarily steps up as a moderator and drives the debate to a conclusion. It could be expected that by combining decision making and appeal processes more clearly and organising debates in a more result-oriented fashion, the tendencies towards bike-shedding and indecision that communities exhibit can be overcome.

\subsection{Community membership, roles and privileges}

The communities apply a broad definition of what makes a community member. "Everybody on the mailing list" who actively participates is considered to be one. Those who contribute more, over an extended period of time, begin to form a loosely defined "core team" early in the process, which also separates those who "merely talk" from those "doing the work". Formal membership in the support organisation forms another community rank. Being appointed to a board or elected leadership position is another one. This suggests a hierarchy of influence that may be misleading, as advancing through the community ranks does not necessarily happen on a straight career path. The differentiation between product contributions and community management may lead to contributors gaining leadership positions that never contributed to the community product. Authority is also gained ad-hoc by individual contributors self-identifying with the initiative to manage a debate, or a community process like writing the manifesto. It can be observed that once contributors reach a board or elected representative level position, they rarely ever go back to being regular contributors. This indicates that such positions do form a sort of end-of-career achievement. Community rank is considered significant in that individuals would, for example, list their community achievements in their CV.

Contributors advance through the community rank meritocracy based on their contributions. Not all contributions are valued the same. Contributors to the core product, founders and individuals "rich on time" advance through the meritocracy more easily. Typically, contributors gain more merit when contributing directly to the community product, as opposed to covering support functions as in helping with administration or event management. Even auxiliary product contributions like the work done by designers and documentation authors are less likely to be appreciated. This may inhibit effective specialisation as the different "professions" within the community carry different merit. The inherent contradiction of the "who does the work decides" rule applied by many communities is that in an advanced community it is almost impossible to identify which specialised task is more important and who does the work.

The phase during which the contributor joins a project also affects the opportunities to advance 
through its meritocracy. Project founders and early contributors often remain in an influential position over a long time. Sometimes they evolve to be "luminaries" or "top dogs" that carry strong influence over community processes and are involved in many community decisions even without a formal role. Long-standing and early contributors aggregated merit that enables them to influence the community as a whole. This poses a difficult barrier for newcomers to become contributors, and even more for existing contributors to advance within the community status groups. The communities are aware of barriers to entry and work to keep them low, It seems however that the barriers are higher, not lower, for advancement and individual personal development within the community.

Many of the interviewed project founders and early contributors who rose to community leadership functions in the early and medium stages of the project emphasise that their motivation was to help the project, not to further their personal reputation. Some say that they were willing to do the work no other contributor wanted to do. Others stress that the perception of the president's position is of much higher value to others than to them. Their expectation towards other community leaders is that they would also mainly work towards the interests of the community, not their own benefit. Some interviewees admitted that other contributors might regard the group of founders and early contributors as a "round table" that is a bit out of touch with the rest of the community. The modesty expressed by the founders and early community leaders is convincing in the early and medium stages of community growth. For late stage communities, it must be assumed that the prestige and remuneration for serving in a community leadership role becomes an attraction in itself. Late stage communities will then require a system of checks and balances to maintain control over community management, which was unnecessary and therefore not established in the early and medium stages.

Similar to organisational structure there is an implicitness in the community status groups, roles and privileges. The self-referential authority within the community is well-understood by the founders and early contributors. One interviewee said, "I set the rules once, I can do it again." This freedom to question rules and apply norms where they are applicable and ignore or bend them otherwise is second nature to old-timers. It is explicitly communicated, as in "if a rule prevents you from improving or maintaining Wikipedia, ignore it", but difficult to grasp for newcomers. Rules solidify, sometimes unwantedly, and are rarely ever changed for a late stage community. The open doors policy that the communities are proud of deteriorates in the late stage. Long-standing administrators expressed worries that "some of us have lost the trust that newcomers will do good things".

\subsection{Structural reforms and organisational design}

In all three cases, the explicit and implicit organisational structure emerged in the early and midstages of community development. While the initial structure developed implicitly, the communities did create well-working formal supportive organisations that originally served their purpose well. However, they did not implement a systematic and periodic review process which ensures that implicit and explicit structure and processes do not diverge too much, and that the formal organisation stays focused on the mission that the community created it for. They also implemented partially insufficient checks and balances to enforce accountability of these organisations towards their contributor base. Through membership open to all active contributors and direct as well as competitive elections of community representatives by the members, KDE e.V. remained most effective and accountable to the community of the three cases.

With the removal of the elected fellowship representatives and the position of the executive director, FSFE grew less accountable in 2018. While it does still represent the ideals of software freedom and aims to speak for the wider Open Source community, it gains few new contributors.

Of the three cases, Wikimedia Deutschland e.V. developed to be most removed from its original purpose of serving the German language Wikipedia community. While it drives fundraisers and shares the name of the community project, most of its activities and most of its budget do not 
directly support the community of authors. While all its activities are charitable and contribute to the cause of free knowledge, almost all they share with the community of authors is the name. It is reasonable to assume that this fact contributes to the declining number of authors, as potential contributors realise that their work is being used to raise funds for mostly unrelated activities and a large body of staff.

All communities exhibit an aversion against administrative processes or "bureaucracy", resulting in an apparent lack of momentum towards active organisational change. Interviewees from all three communities mentioned that formalising Structure and documenting, decision making and conflict resolution processes to a necessary extent helps to maintain the freedom to participate and joy of contribution. They face the challenge of preserving "hacker culture" while at the same time enabling large numbers of contributors to collaborate successfully.

Based on the lack of organisational design, community processes and structures are largely implicit and there are no well-defined rules of appeal. The German-speaking community of Wikipedia authors provides a positive example of well-working auto-organisation. However, these processes are independent of Wikimedia Deutschland e.V. as their support organisation, indicating that the resulting lack of accountability offers opportunities for self-serving behaviour.

This raises the question of how communities can ensure that their structure and processes evolve so that they continue to fulfil their mission of supporting their contributors. Where competition keeps businesses aligned with their purpose and elections align the actions of politicians with the interests of the population, FOSS communities depend on voluntary participation to raise contributions. This postulates the number of independent contributing entities and the number of contributions raised as key metrics for community health. Implicit and explicit community structure and processes should primarily aim to support these goals. Community activities, also by the support organisations, should be assessed based on how they contribute to these goals. From the budget a community is raising, every Euro that is spent on activities that do not contribute to these goals reduces the number of attracted contributors and through that the potential impact and success of the community. The selfreferential purpose of FOSS communities means that all functions of the community need to be accountable to the base of its active contributors. In turn, a community can only represent those that by way of actively contributing acquire an equal voice in decision making and conflict resolution processes.

\section{Summary}

This study started out from the observation that FOSS communities struggle to maintain growth once they reach a large number of contributors. It could be observed that the growth phases the communities proceed through can be grouped into an initial stage with ad-hoc coordination and an equivalence of individual and group goals, a middle stage of growth with consensus-focused autoorganisation and a late stage with more profound functional differentiation and formal structure.

Businesses, individual volunteers and staff members participate for different sets of reasons. The concept of community composition refers to the mix of volunteers, businesses and staff that engage in a community. Assuming that, all things being equal, governance norms develop depending on community composition. The study analysed three primarily volunteer driven communities to provide an insight into their governance and to identify commonalities between them even though they create vastly different products. Based on the principle of voluntary participation, the purpose of communities is defined in a self-referential manner: The community serves the interests of the contributors that form it, with no outside authority except the law. This means communities need to solve the constitution problem to define who has a voice and to establish structure as well as decision making and conflict resolution processes, based on voluntary participation. 
Community governance is shaped by the mindset of the contributors. Individual volunteers are primarily intrinsically motivated individuals, which is reflected in the expectations they expressed in the interviews: motivated to participate in a community of makers, to experience equality of opportunity among their peers, to find a balance between makers and community builders, to become a part of an ambitious, productive meritocracy and to see their own ethical principles represented in the community governance norms. If these expectations are fulfilled, they develop increasing loyalty towards the community.

The case studies reflected the concepts of membership, the formal and informal organisational structure and the decision making and conflict resolution processes of the communities against these expectations. They indicate that while there is a close or close enough match in the initial and middle stages, in particular the formal organisations that have been created to support the work of the community show a tendency of distancing themselves from the community goals. The combination of solidified implicit norms and more closed-up organisations creates barriers to entry for newcomers and reduces the number of long-term, loyal contributors the community is able to attract in the late stage. While it remains relatively easy to contribute to the community product, it becomes increasingly hard to gain access to influential formal roles and positions.

The gap between makers and community builders grows with size of the community. Independent of the effort invested in setting up the original support structure, the formal organisations partly disconnect from their communities. This seems to be caused by the absence of a regular review process based on checks and balances built into community governance, resulting in a lack of accountability of the support organisations towards their communities.

Volunteer contributors exhibit aversion to authority and formal decision making. At the same time, they jointly form the highest authority within their community. A possible conclusion is that community decision making processes should be well-defined, and that the highest level of escalation should be the community as a whole. Conflict resolution mechanisms should mirror the decisionmaking processes.

The communities investigated in this study partially lacked instruments to ensure that their structure and processes supported the overall community goals. Similar to elections in politics and supervisory boards representing investor interests in enterprises, communities need to re-align decision making power and require accountability to remain volunteer driven in a successful transition into the late stage of community growth.

\section{About the author}

Mirko Boehm is a Free and Open Source Software contributor, primarily as a developer and speaker. He is the founder of the Quartermaster project, and has been a contributor to major Open Source projects like the KDE Desktop since 1997, including several years on the KDE e.V. board. He is a visiting lecturer and researcher on Free and Open Source Software at the Technical University of Berlin, part of the FSFE Germany team and a Qt-certified specialist and trainer. Mirko Boehm has a wide range of experience as an entrepreneur, corporate manager, software developer and German Air Force officer.

The Open Invention Network protects the Open Source ecosystem by acquiring patents and licensing them royalty free to all participants. As director for the linux system definition, Mirko Boehm is responsible for the technical scope that defines the field of use of the patent non-aggression agreements. 
As a co-founder of Endocode, an employee-owned, shareholder company based in Berlin, Germany providing professional IT services with a focus on Open Source technologies, Mirko Boehm specialises in consulting to and mentoring startups and medium to large businesses. His areas of expertise include complex software development endeavours, the use of Open Source products and methods in organisations, and technology related issues of business strategy and intellectual property.

He lives with his wife, two kids and two cats in Berlin.

\section{Licence and Attribution}

This paper was published in the Journal of Open Law, Technology, \& Society, Volume 11, Issue 1 (2019). It originally appeared online at http://www.jolts.world

This article should be cited as follows:

Boehm, Mirko (2019) 'The emergence of governance norms in volunteer-driven open source communities', Journal of Open Law, Technology, \& Society, 11(1), pp 3 - 39 DOI: $10.5033 /$ jolts.v11i1.131

Copyright (C) 2019 Mirko Boehm.

This article is licensed under a Creative Commons Attribution 4.0 CC-BY available at https://creativecommons.org/licenses/by/4.0/

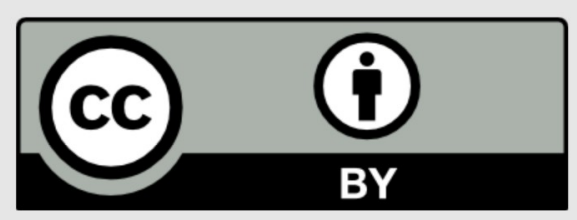

